Article Published: 08 March 2018

\title{
Depression promotes prostate cancer invasion and metastasis via a sympathetic-cAMP-FAK signaling pathway
}

Yan Cheng, Xing-Hua Gao, Xian-Jing Li, Qiu-Hua Cao, Dan-Dan Zhao, Jin-Rong Zhou, Hong-Xi Wu, Yun Wang, Lin-Jun You, Hong-Bao Yang, Yun-Long He, Yong-Ren Li, Jin-Song Bian, Qing-Yi Zhu, Lutz Birnbaumer $\square \&$ Yong Yang $\square$

Oncogene 37, 2953-2966(2018)

$\mathbf{8 0 2}$ Accesses $\mid \mathbf{3}$ Citations $\mid \mathbf{8}$ Altmetric $\mid$ Metrics

\section{Abstract}

Depression drives cancer progression and induces poor clinical outcome. However, the mechanisms underlying depression and cancer outcomes are unclear. In this work, we investigated 98 prostate cancer patients and found that patients with high score of psychological depression were correlated with tumor invasion and metastasis. We found focal adhesion kinase (FAK) was increased in cancer patients with metastatic features and high score of depression. FAK knockdown completely blocked depression-promoted tumor invasion in orthotopic transplantation tumors. In Hi-myc mice and a murine model of depression, sympathetic activation was detected in the prostate tissue. Further we showed that FAK activation was dependent on a cAMP-PKA signaling pathway. Our results demonstrated that the activation of a sympathetic-FAK signaling pathway in prostate cancer patients with high degrees of depression facilitates tumor invasion. We suggest that blocking $\beta 2 \mathrm{AR}$ with propranolol or inhibiting FAK activation with PF562 271 may be novel strategies for depressed patients with invasive prostate cancer.

\section{Introduction}


Increasing number of cancer patients are found to experience a depressed mood or a loss of interest in daily activities for a long time [1,2]. Although epidemiologic studies have shown that psychological depression is related to cancer mortality $[3,4,5]$, the biological pathways and precise mechanisms linking depression and tumor progression lack experimental evidence. Basic research on neuro-oncology has shown that nerve densities are in positive correlation with prostate tumor grades [6]. Of note, prostate cancer occurred more frequently and was significantly greater in the peripheral zone than that of the transition zone possibly because of the high nerve density in the peripheral zone [7]. A recent study observed that genes in two key pathways, the adrenergic and glucocorticoid signaling pathways, exhibit dysregulation in the tumors of lethal prostate cancer in comparison with nonlethal prostate cancer [8]. These two pathways are also activated during depression [9]. Thus, identifying the nerve density and the precise neuroendocrine signaling in depression are critical for better understanding the mechanisms linking depression and prostate cancer progression.

Patients with organ-confined prostate cancer are usually successfully treated. However, recurrence of the disease often exhibits metastasis, which is virtually incurable and results in significant disease mortality $[10,11]$. Cell invasion across basement membrane barriers is a critical step of prostate cancer metastasis $[12,13]$. Alteration of the extracellular matrix $(\mathrm{ECM})$ is increasingly found to be important in facilitating tumor invasion $[14,15]$. Recently, several lines of evidence have shown a close association between stromal gene expression and invasive phenotype of primary prostate cancer [16]. Among them, focal adhesion kinase (FAK, gene name PTK2), a key regulator in the focal adhesions, has been focused on because it can be recruited to sites of integrin clustering and regulate cell motility through cytoskeletal rearrangement [17]. In prostate cancer, FAK has been reported to promote neuroendocrine differentiation in the Transgenic Adenocarcinoma of the Mouse Prostate (TRAMP) model [18]. However, the overall significance and the molecular mechanisms through which depression induces prostate cancer invasion and whether the depression-induced FAK activation plays a role in prostate cancer invasion remain uncertain.

In this study, we found that depression was correlated with increased prostate cancer invasion and 
metastasis as well as elevated FAK levels in clinical cancer tissues. Knockdown of FAK blocked depression-induced prostate cancer invasion and metastasis. More importantly, we demonstrated for the first time that depression increased sympathetic nerve infiltration into the prostate. Elevated norepinephrine activates FAK by the $\beta 2$ AR-cAMP-PKA signaling pathway. Furthermore, we found that norepinephrine-induced FAK activation increases the ability of cell movement via paxillin phosphorylation and regulates ECM remodeling via matrix metalloproteinase (MMP) release, both facilitating tumor invasion. Therefore, inhibition of the depression-activated sympathetic-FAK signaling pathway by the 32 AR blocker propranolol or the FAK inhibitor PF562 271 should be beneficial for prostate cancer treatments, especially for clinically depressed patients with invasive prostate cancer.

\section{Results}

\section{FAK expression and phosphorylation are correlated with high symptoms of psychological depression and metastatic features in prostate cancer patients}

To determine the role of depression in prostate biology, a total of 98 patients with prostate cancer were included in this study. We initially grouped patients into high and low degrees of depression based on the PHQ-9 and the Hamilton Depression Scales [19, 20]. We found that high scores in the Gleason and TNM staging systems, showing presence of vascular invasion plus lymph node and/or bone metastasis correlated significantly with high degrees of depression. However, depression had no relevance to age, tumor volume or serum PSA levels (Supplementary Table 1). These results suggest that depression is clinically important in prostate cancer invasion and metastasis.

To identify the mechanisms underlying how depression impacts prostate cancer invasion and metastasis, we searched the GEO databases and found only one database containing samples of cancer patients divided by low and high depression levels (GSE9116). Gene set enrichment analysis (GSEA) showed highly enriched genes relevant to cell adhesion molecules and ECM receptor interaction (Fig. 1a). Furthermore, we searched for the expression of the hub molecules in The Cancer Genome Atlas (TCGA) containing prostate cancer samples with different Gleason score. We found no obvious changes including NRCAM, FBN1, VCAM1, and ICAM2 
(Supplementary Figures 1a-d). But, PTK2 (protein name FAK) expression was continuously elevated when Gleason score increased from 6 to 9 (Fig. 1b). However, it had no changes in patients with low Gleason score (GSE5132) (Fig. 1c). In addition, PTK2 expression was highly elevated in metastatic prostate cancer compared with that in adjacent or localized prostate cancer (GSE3325 and GSE6919) (Fig. 1d, e). 
Fig. 1

a

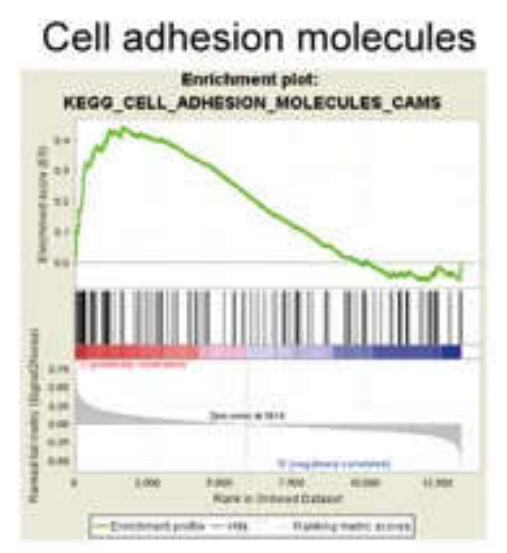

C

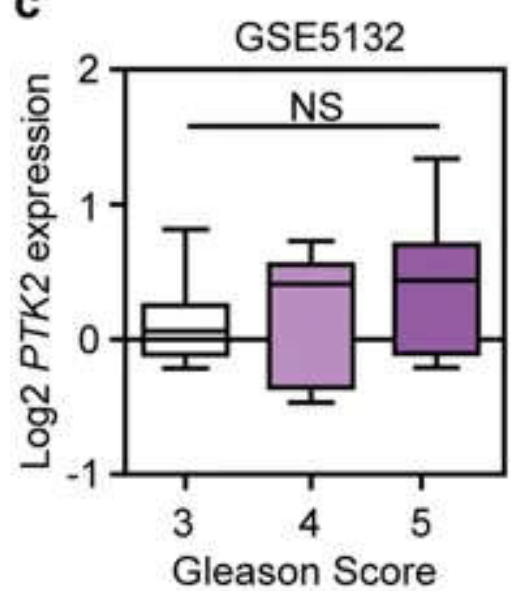

GSE9116

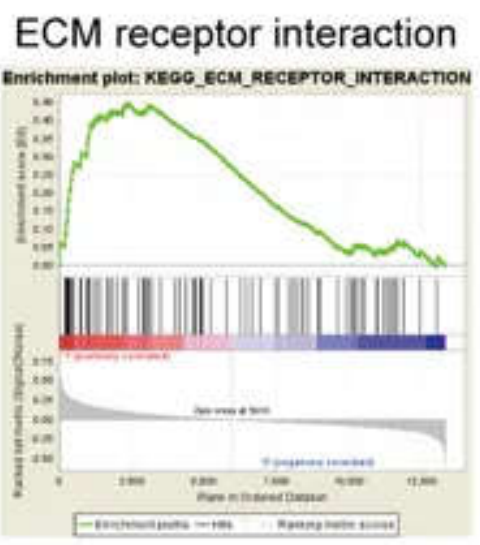

d

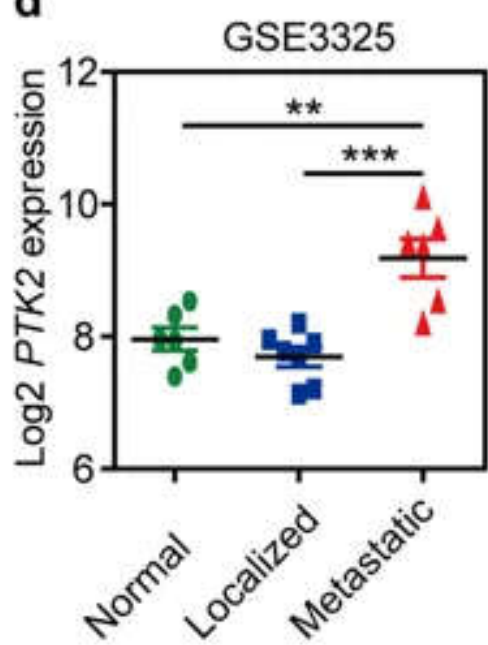

b

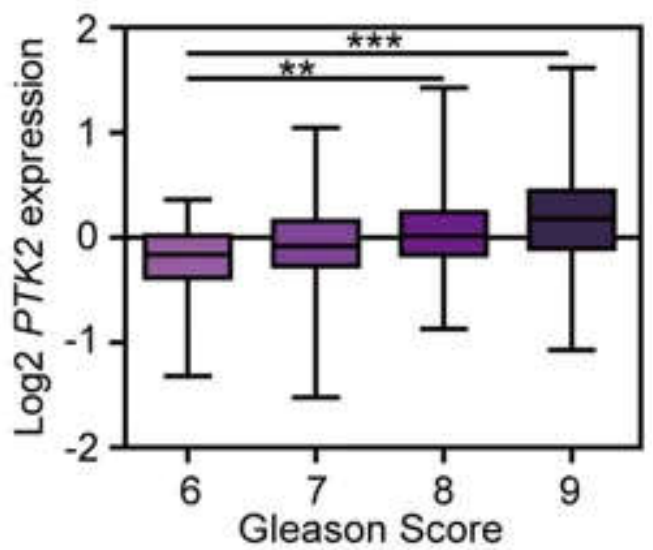

e

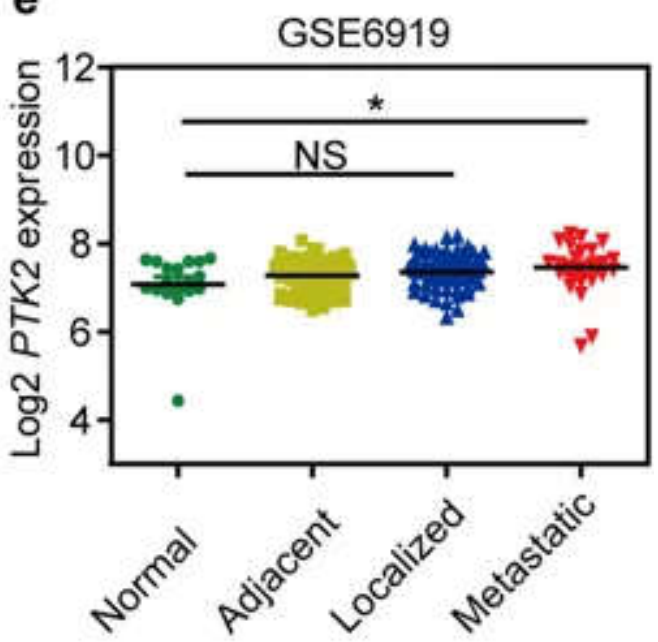

f

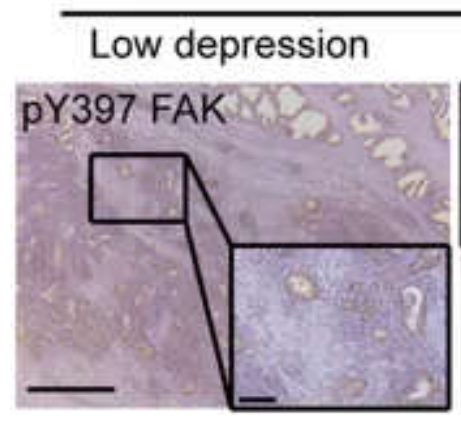

Gleason Score 6 - 7 High depression

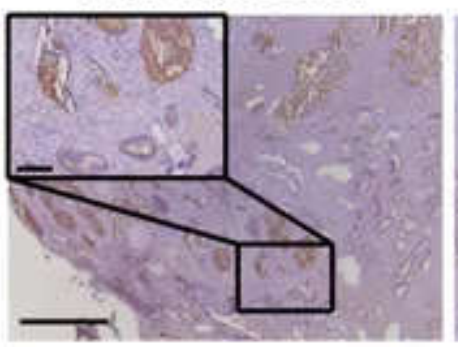

Gleason Score 8-10

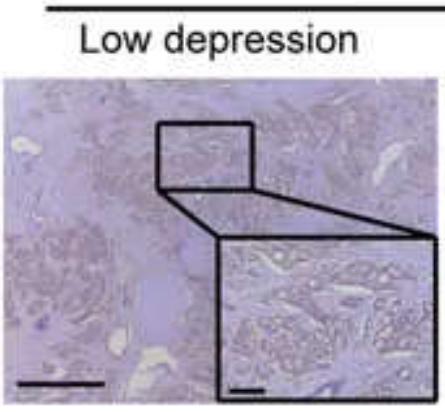

High depression

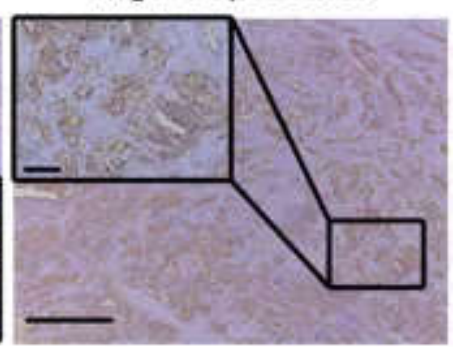

FAK is highly expressed and phosphorylated in prostate cancer patients with high degrees of depression and high metastatic features. a Gene set enrichment analysis (GSEA) plot of cell adhesion molecules (NES $=1.78$; Nominal $P$-value $=0.0 ;$ FDR $q$-value $=0.017$ ) and ECM receptor interaction (NES $=1.73$; Nominal $P$-value $=0.0 ;$ FDR $q$-value $=0.028$ ) gene sets in GSE9116. $\mathbf{b}$ Relative expression of PTK2 mRNA in prostate cancer patients with high Gleason score in the TCGA prostate cancer database ( $n=44$ in Gleason score 6 group; $n=237$ in Gleason score 7 
group; $n=60$ in Gleason score 8 group; $n=128$ in Gleason score 9 group). c Relative expression of PTK2 mRNA in prostate cancer patients with high Gleason score in GSE5132 database $(n=11$ in Gleason score 3 group; $n=12$ in Gleason score 4 group; $n=8$ in Gleason score 5 group). $\mathbf{d}$ Relative expression of PTK2 mRNA in metastatic prostate cancer patients in GSE3325 datasets ( $n$ $=6$ in Normal group; $n=7$ in Localized group; $n=6$ in Metastatic group). e Relative expression of PTK2 mRNA in metastatic prostate cancer patients in GSE6919 datasets ( $n=18$ in Normal group; $n=67$ in Adjacent group; $n=61$ in Localized group; $n=25$ in Metastatic group). $\mathbf{f}$ Representative images of FAK phosphorylation in prostate cancer patients with high and low degrees of depression. Bar: $500 \mu \mathrm{m}$ (low magnification); $100 \mu \mathrm{m}$ (high magnification). The statistical analysis was based on one-way ANOVA with Tukey's post-hoc tests ( ${ }^{\star} P<0.05 ;{ }^{* \star} P<0.01 ;{ }^{* \star *} P<0.001$ )

Next, we examined the expression of FAK in prostate patients with depression. We found FAK phosphorylation (Tyr397) was significantly higher in prostate cancer patients with high depression compared with that in patients with low depression within the same Gleason score (Fig. 1f). Altogether, our clinical data supports the possibility that FAK is the key molecular connector linking depression and prostate cancer invasion and metastasis.

\section{Inhibition of FAK blocks depression-promoted tumor invasion and metastasis in orthotopic prostate cancer models}

We hypothesized that FAK mediates depression-promoted prostate cancer invasion and metastasis. To test this hypothesis, we established FAK knockdown PC-3 and 22Rv1 cell lines (Supplementary Figures 2a-c), and then we used bioluminescent xenograft models to study the role of FAK in depression-promoted prostate tumor invasion and metastasis in vivo (Fig. 2a). We first tested the $22 \mathrm{Rv} 1$ prostate tumor model and found that restraint stress significantly decreased body weight in both shNC and shPTK2 groups (Fig. 2b) and the mice exhibited depressive behavior shown by decreased sucrose preference in the two stressed groups (Fig. 2c). Using bioluminescence imaging, we found that stress/depression significantly increased orthotopic prostate tumor invasion and mesentery metastasis in shNC groups 5 weeks after tumor implantation. However, FAK knockdown completely abrogated depression-promoted tumor invasion and metastasis (Fig. 2d, f). Similar results were obtained in the PC-3 prostate tumor model (Supplementary Figures 3a and b). 
Hematoxylin and eosin (H\&E) staining and Masson staining showed an intact capsule of the orthotopic transplantation prostate tumor in non-stressed groups. However, prostate tumor in depressed mice broke out of the capsule and invaded the prostate stroma with increased collagens. As expected, these effects were abolished in mice implanted with shPTK2 prostate cells (Fig. 2g). Thus, FAK is critical for depression-promoted invasion of prostate cancer. 


\section{Fig. 2}
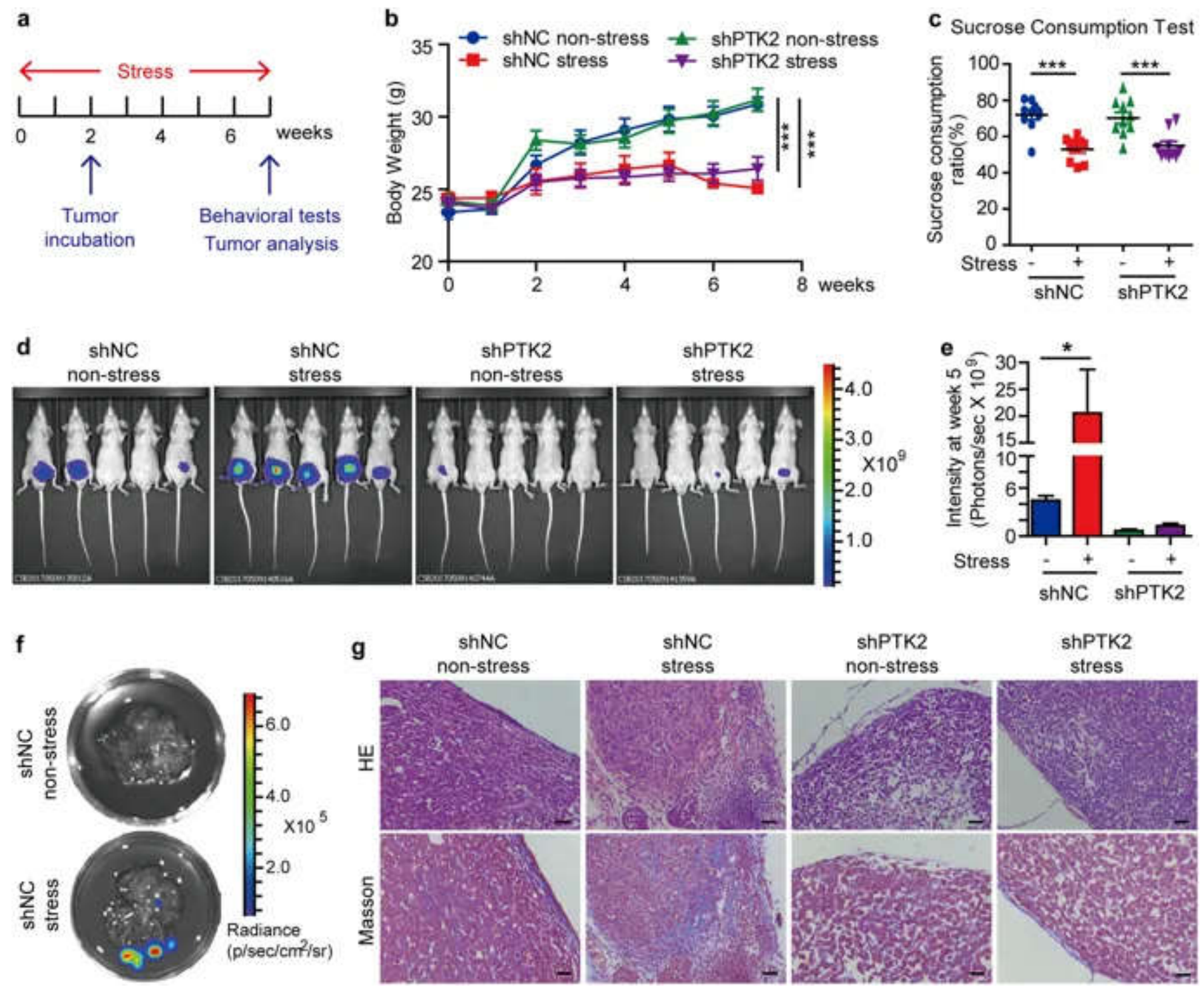

FAK blockade prevents psychological depression-promoted tumor metastasis and desmolpasia in the orthotropic $22 R v 1$ model. a Time course of restraint stress and tumor implantation. b Body weight of mice $(n=10)$. The statistical analysis was based on Two-way ANOVA with Bonferroni posttests (shNC non-stress vs shNC stress, from week 4 to week 7 , ${ }^{* * *} P<0.001$; shPTK2 nonstress vs shPTK2 stress, from week 4 to week $\left.7,{ }^{* \star *} P<0.001\right)$. c Sucrose consumption ratio in the sucrose preference test $(n=10)$. The statistical analysis was based on One-way ANOVA with Tukey's post-hoc tests ( ${ }^{* \star *} P<0.001$ ). $\mathbf{d}$ Illustrative examples of in vivo bioluminescence imaging of 22Rv1 cells orthotropic implanted in the ventral prostate of nu/nu mice $(n=5)$. e ROI analysis of fluorescence intensities from the 22Rv1 cells orthotropic tumors $(n=10)$. The statistical analysis was based on One-way ANOVA with Tukey's post-hoc tests $\left({ }^{\star} P<0.05\right)$. f Representative fluorescence images of metastatic sites in the 22Rv1 model. $\mathbf{g} \mathrm{H} \& \mathrm{E}$ and Masson staining of the 
prostate in the $22 \mathrm{Rv} 1$ model. Bar: $50 \mu \mathrm{m}$

\section{Depression promotes prostate cancer invasion in Hi-myc mice}

We explored the molecular mechanisms by which FAK mediates prostate cancer invasion in depression. First, we generated a depression model by applying chronic unpredictable mild stress (CUMS), which has been widely used as an animal model of depression [21,22,23] to Hi-myc mice (Fig. 3a). Due to the long latency of Hi-myc mice to develop invasive cancer, we modified the CUMS protocol with longer time duration and milder daily stressors (Supplementary Table 2). We found that both wild-type (WT) and Hi-myc mice exhibited depressive behavior after 12 weeks of stress (Fig. 3b, c). Animals were sacrificed at 24-weeks of age, a time sufficient to allow the invasive growth of prostate cancer. We observed that prostate weights in stressed Hi-myc mice were significantly higher than that in non-stressed Hi-myc mice (Fig. 3d). Interestingly, we found that the dorsolateral prostate lobe (DLP), ventral prostate lobe (VP), and anterior prostate lobe (AP) were adhered to each other and difficult to separate in stressed Hi-myc mice, whereas clear boundaries were observed in non-stressed Hi-myc mice. Further experiments with H\&E staining and immunohistochemical staining of $\alpha$-SMA showed that the tumor was mostly restricted to the smooth muscle in non-stressed Hi-myc mice. Strikingly, the tumor was more invasive in stressed Hi-myc mice shown by breaking through the basement membrane and infiltrating into the stroma (Fig. 3e, f). Taken together, these findings reveal that depression promotes prostate tumor invasion in Hi-myc mice. 


\section{Fig. 3}

a Hi-Myc tumor progression and experimental time line

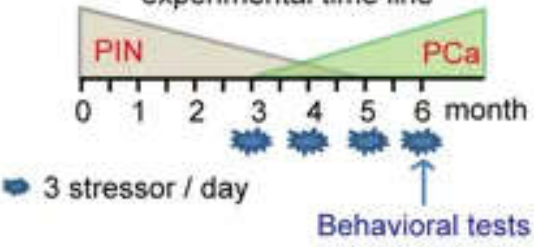

Tumor analysis d

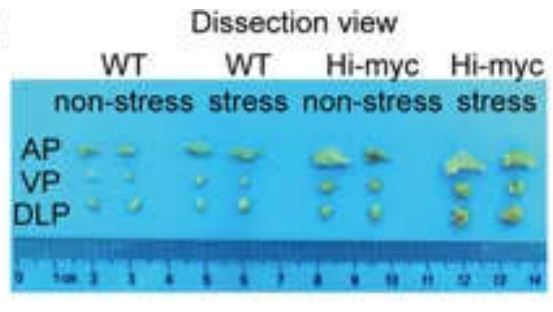

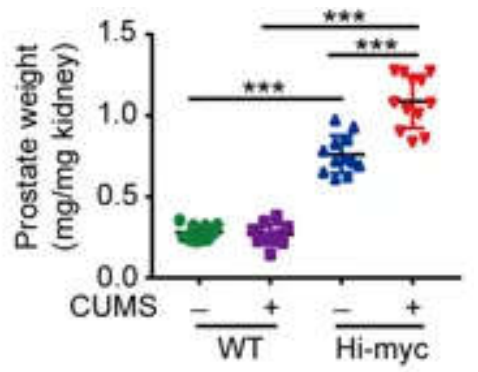

b

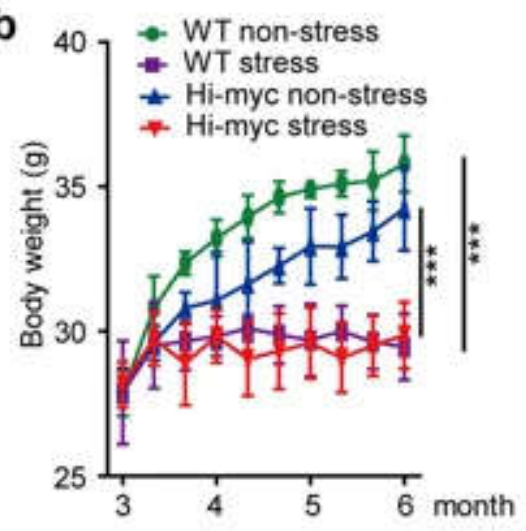

C

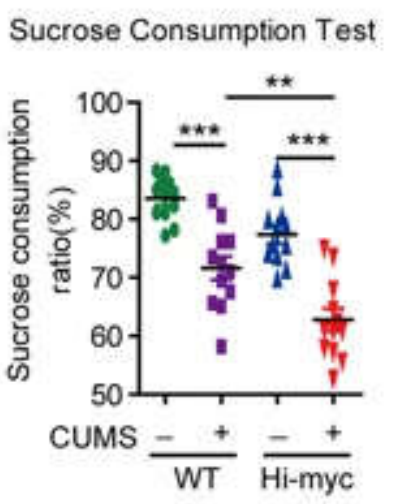

Foced Swimming Test

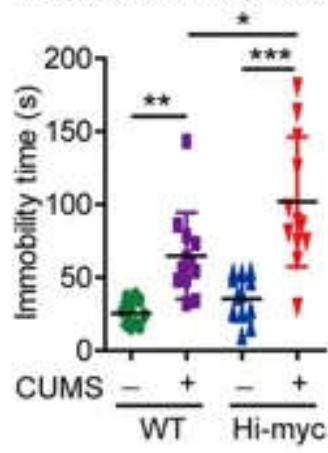

Tail Suspension Test

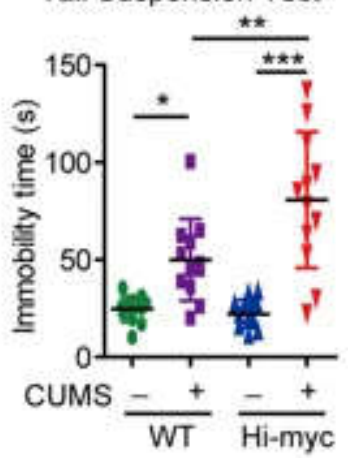

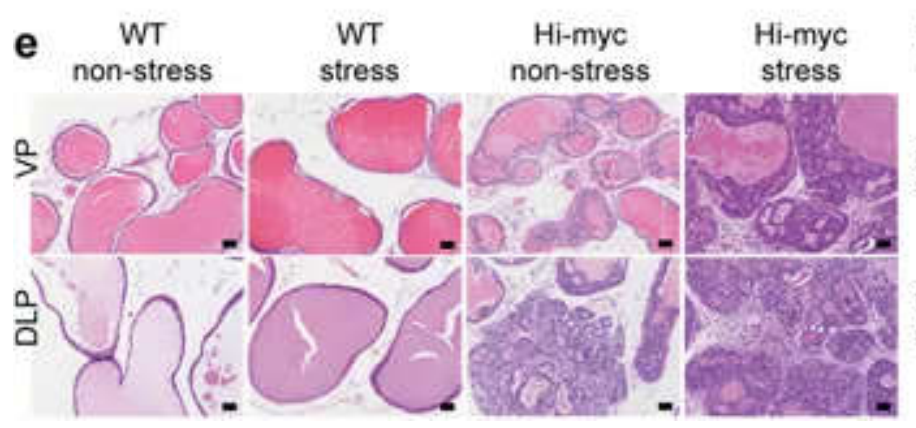

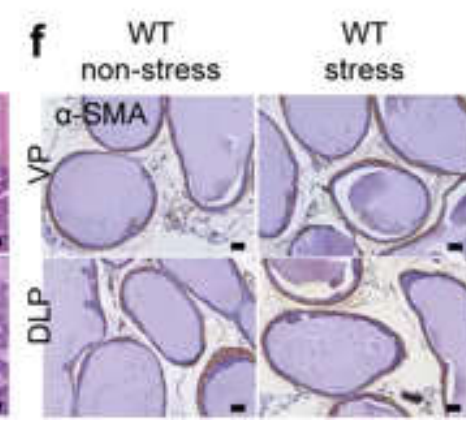

$\mathrm{Hi}$-myc non-stress

Hi-myc stress

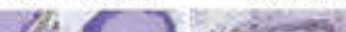

Depression promotes prostate cancer invasion in Hi-myc mice. a Timeline for Hi-myc mice tumor progression and experimental protocols. 3-month-old wide type (WT) and Hi-myc mice were exposed to chronic unpredictable mild stress (CUMS) for six months, the behavioral monitoring was measured at the end of the experiment, mice were sacrificed when 6-month-old. b Body weight of mice $(n=12)$. The statistical analysis was based on Two-way ANOVA with Bonferroni posttests (WT non-stress vs WT stress, from month 5 to month 6 , ${ }^{* * *} P<0.001$; Hi-myc non-stress vs Hi-myc stress, from month 4 to month $\left.6,{ }^{* * \star} P<0.001\right)$. c Behavioral tests at the end of CUMS treatment. Sucrose consumption ratio in the sucrose preference test $(n=12)$; Immobility time in the forced swimming test $(n=12)$ and Immobility time in the tail suspension test $(n=12)$. The statistical analysis was based on One-way ANOVA with Tukey's post-hoc tests $\left({ }^{\star} P<0.05\right.$; ${ }^{* \star} P<$ $\left.0.01 ;{ }^{* \star} P<0.001\right)$. d Left: Representative macroscopic images of dissected mouse anterior, ventral, and dorsolateral prostate lobes (AP, VP, and DLP lobes); Right: Wet weight of total prostate was expressed as $\mathrm{mg} / \mathrm{mg}$ kidney weight $(n=12)$. The statistical analysis was based on 
One-way ANOVA with Tukey's post-hoc tests ( ${ }^{\star * * P} P<0.001$ ). e H\&E staining of AP, VP, and DLP lobes $(n=3)$. Bar: $50 \mu \mathrm{m}$. f Immunohistochemical staining of $\alpha$-SMA in VP and DLP $(n=3)$. Bar: 50 $\mu \mathrm{m}$. WT wild type, PIN prostatic intraepithelial neoplasia, PCa prostate cancer, AP anterior prostate, VP ventral prostate, DLP dorsolateral prostate, stress CUMS, chronic unpredictable mild stress

\section{Depression activates FAK and induces an aligned desmoplastic ECM in Hi-myc mice}

Next, we examined the role of depression in FAK expression and phosphorylation in Hi-myc mice. We observed significant up-regulation of FAK mRNA, protein and its phosphorylation in stressed Hi-myc mice compared with non-stressed Hi-myc mice (Fig. 4a-c). It has been reported that FAK phosphorylation increases MMPs via ERK activation, which are the key regulators in reforming ECM and play an essential role in tumor invasion [14]. So, we examined the expression of MMP-2 and MMP-9 by qPCR and gelatin zymography. We found both the mRNA and the active forms of MMP-2 and MMP-9 were significantly increased in stressed Hi-myc mice (Fig. 4d, e). In further support of depression-induced FAK activation promoting prostate cancer invasion via MMPs, we looked at the characteristics of ECM by Masson's trichrome staining. We found abundant collagens in the ECM of Hi-myc mice compared with WT mice. More importantly, stress increased collagens, and remolded the ECM into a more thick and straight form (Fig. 4f). In line with this, we observed increased expression of the collagen-associated genes $\operatorname{ctg} f$ and $\operatorname{tg} f b l$ in the stressed Hi-myc mice (Fig. 4g, h). Thus, depression activates FAK and promotes prostate cancer invasion via ECM remodeling. 
Fig. 4

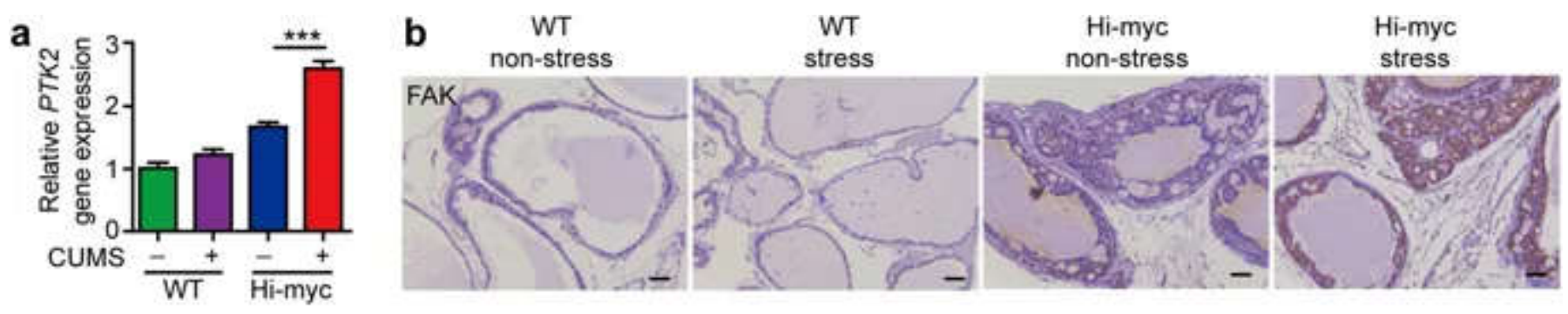

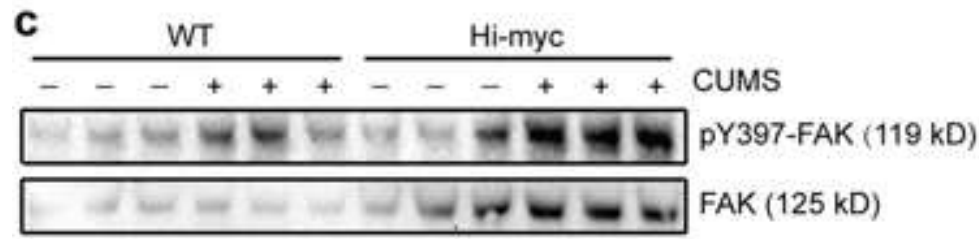

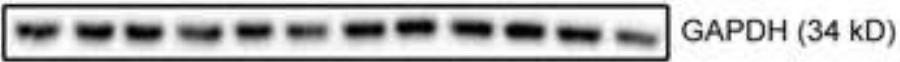

d

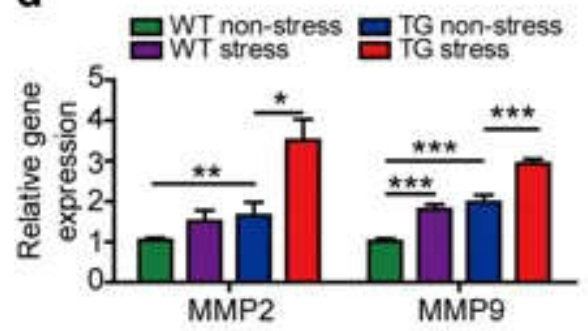

e

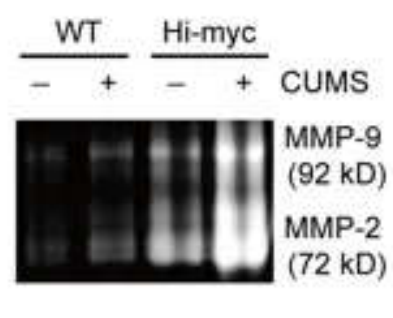

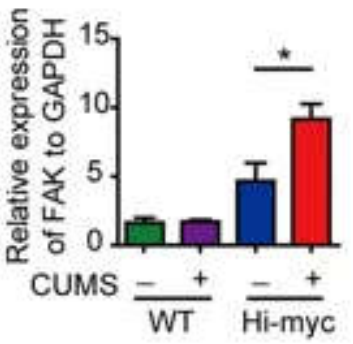

g

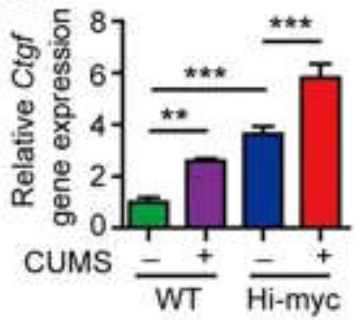

Hi-myc stress

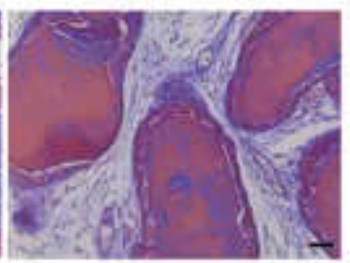

Hi-myc stress

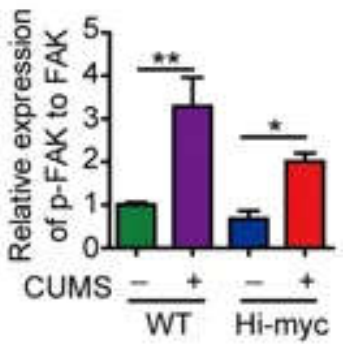

h

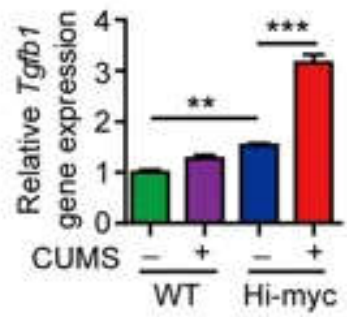

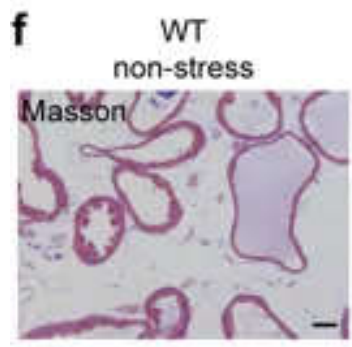

stress non-stress
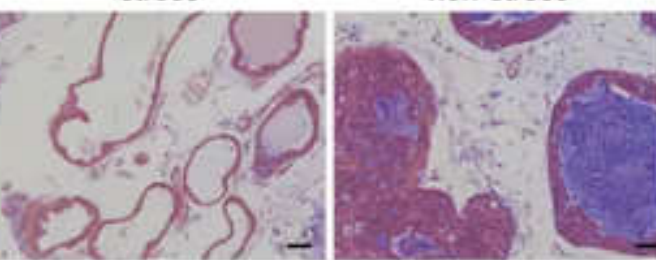

Hi-myc

non-stress
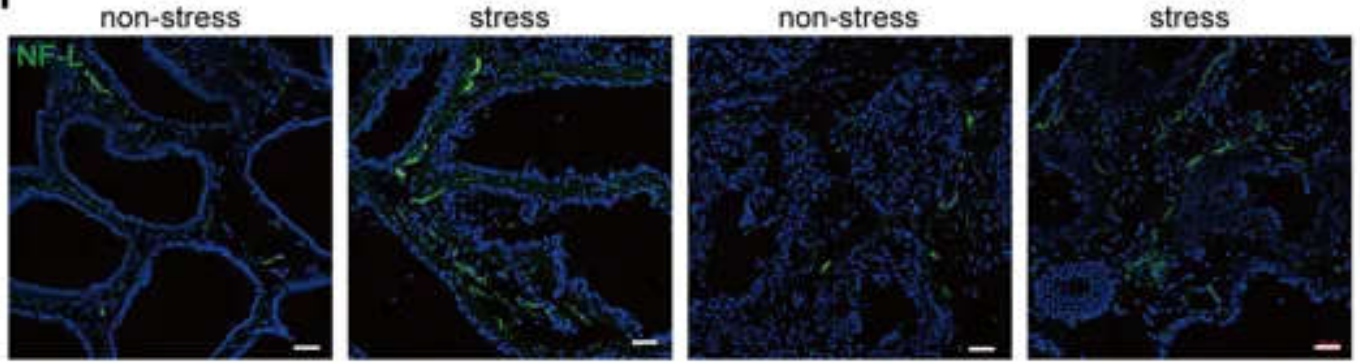

Depression activates FAK and induces an aligned desmoplastic ECM in Hi-myc mice. a PTK2 mRNA assessed by quantitative polymerase chain reaction (qRT-PCR) in the prostate, relative to control group $(n=6)$. The statistical analysis was based on One-way ANOVA with Tukey's posthoc tests ( $\left.{ }^{* \star} P<0.001\right)$. b Immunohistochemistry staining of FAK in the prostate. Bar: $50 \mu \mathrm{m}$. c 
Left: Western blot analysis of pY397 FAK and FAK in the prostate; Right: Quantification of FAK band intensity relative to GAPDH, and pY397 FAK band intensity relative to total FAK $(n=6)$. The results were representative of two independent experiments. ${ }^{\star} P<0.05$, ${ }^{*} P<0.01$, based on Oneway ANOVA with Tukey's post-hoc tests. d MMP9 and MMP2 mRNA assessed by qRT-PCR in the prostate, relative to control group $(n=6)$. The statistical analysis was based on One-way ANOVA with Tukey's post-hoc tests ( $\left.{ }^{\star} P<0.05,{ }^{* \star} P<0.01,{ }^{* * *} P<0.001\right)$. e Zymography for gelatinase activities in the prostate $(n=3)$. The results were representative of three independent experiments. f Masson staining of the prostate $(n=3)$. Bar: $50 \mu \mathrm{m}$. $\mathbf{g}$ Connective tissue growth factor (ctgf) mRNA assessed by qRT-PCR in the prostate, relative to control group $(n=6)$. The statistical analysis was based on One-way ANOVA with Tukey's post-hoc tests ( ${ }^{* *} P<0.01,{ }^{* \star *} P<$ 0.001). $\mathbf{h}$ Transgenic growth factor beta 1 (tgfb1) mRNA assessed by qRT-PCR in the prostate, relative to control group $(n=6)$. The statistical analysis was based on One-way ANOVA with Tukey's post-hoc tests ( ${ }^{\star \star} P<0.01,{ }^{* \star *} P<0.001$ ). i Immunofluorescence staining of NF-L in the prostate. Bar: $50 \mu \mathrm{m}$

\section{Depression increases sympathetic nerve fiber infiltration and elevates norepinephrine levels in Hi- myc mice}

Clinical evidence shows that nerve fiber density in the tumor is correlated to poor clinical outcome [6]. Psychological depression is capable of activating the sympathetic nervous system (SNS) [9]. So, we hypothesized that depression could promote tumor progression and invasion by increasing the nerve fiber densities in prostate tissues. Using immunofluorescence staining of neurofilament- $\mathrm{L}$ (NF-L), we found the number of newly formed nerve fibers was increased in stressed WT and Himyc mice compared with those in non-stressed WT and Hi-myc mice respectively (Fig. 4i). In addition, we quantified the neurotransmitters in the prostate tissues. In agreement with nerve fiber density, we found that norepinephrine was significantly increased in the prostate of both stressed mice. Other neurotransmitters, including dopamine, acetylcholine, and corticosterone, showed no significant statistical difference among the groups (Supplementary Figures 4a-d).

\section{FAK inhibition prevents norepinephrine-induced prostate cancer cell migration and invasion}

Norepinephrine has been reported to be associated with tumor metastasis [24]. A study showed that norepinephrine increased expression of MMP2 and MMP9 in DU145 xenograft tumor tissues [25]. 
To further confirm the role of FAK in depression-promoted prostate cancer invasion and ECM remodeling, we first examined the baseline expression of FAK in prostate cell lines. We found that both FAK mRNA and protein levels were 2-3 fold higher in 22Rv1, DU145, and PC-3 cells than LNCaP cells (Supplementary Figures 5a and b). Next, we used the FAK inhibitor PF562 $2712 \mathrm{~h}$ before norepinephrine treatment. PF562 271 completely abrogated the effects of norepinephrine on PC-3 cell migration and invasion (Fig. 5a, b). Furthermore, shPTK2 also completely blocked norepinephrine-induced PC-3 cell migration and invasion (Fig. 5c, d). Paxillin is a main component of focal adhesions, phosphorylation of which by FAK will lead to the assembly of F-actin fibers for cell movement [26]. We found norepinephrine significantly increased the phosphorylation of paxillin (Tyr118) and enhanced F-actin reassembly in PC-3 cells transfected with shNC.

Downregulation of FAK by shPTK2 completely abrogated theses effects (Fig. 5e). In addition, we observed that norepinephrine significantly increased FAK, paxillin, ERK phosphorylation, and $M M P 2, M M P 9$ mRNA levels in PC-3 cells transfected with shNC, but not in shPTK2 PC-3 cells (Fig. 5f, g). Altogether, these results suggest that FAK is a key molecule for norepinephrineinduced prostate cancer cell migration and invasion. Knockdown of FAK not only abrogated norepinephrine-induced paxillin phosphorylation and cell skeleton remodeling, but also eliminated MMPs. We next assessed whether p-paxillin and MMP levels changed in clinical samples. We found both p-paxillin and MMP levels increased in patients with high degrees of depression (Supplementary Figure 6a). 


\section{Fig. 5}
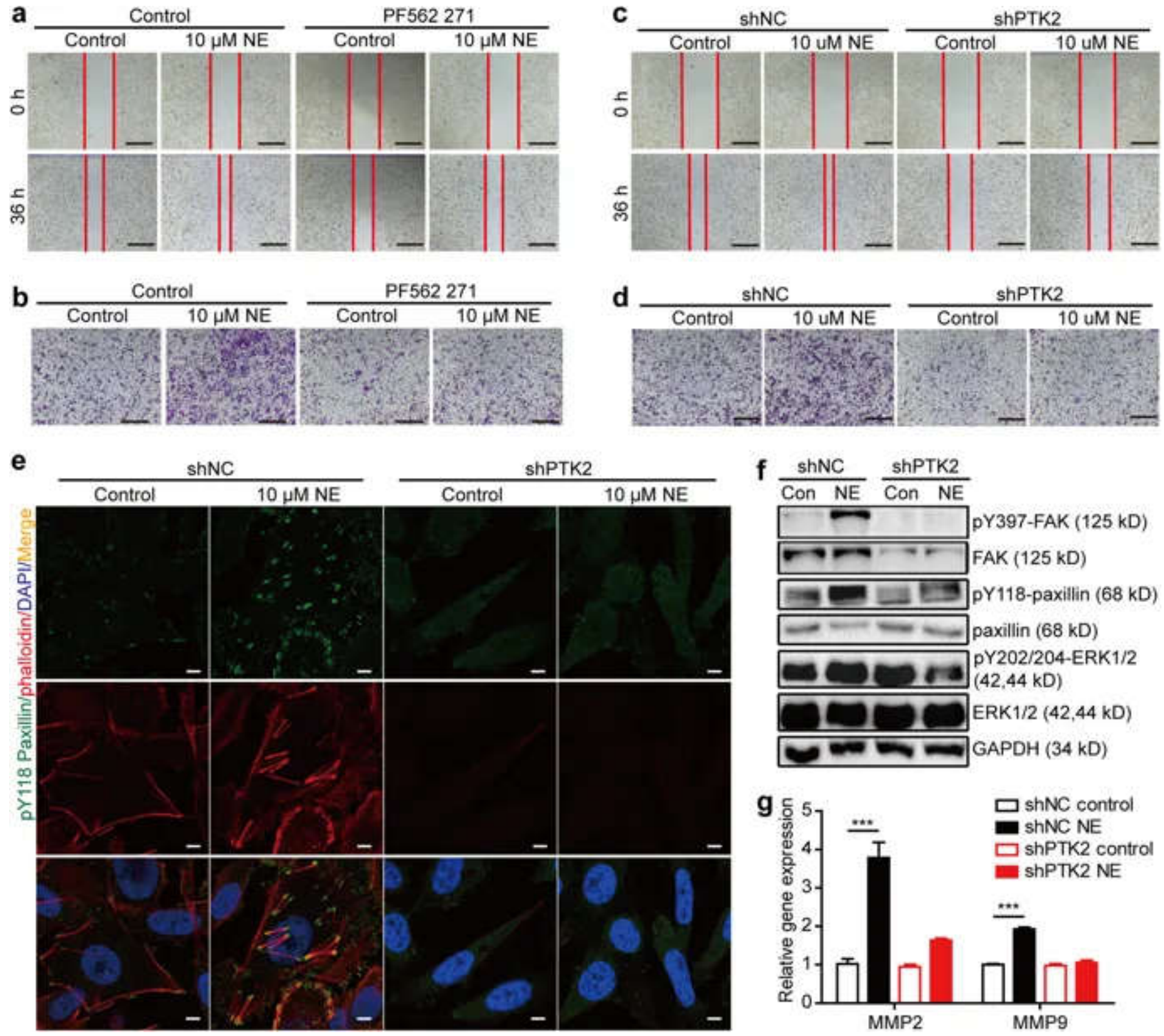

f $\frac{\text { shNC }}{\operatorname{Con} \mathrm{NE}} \frac{\text { shPTK2 }}{\operatorname{Con} \mathrm{NE}}$
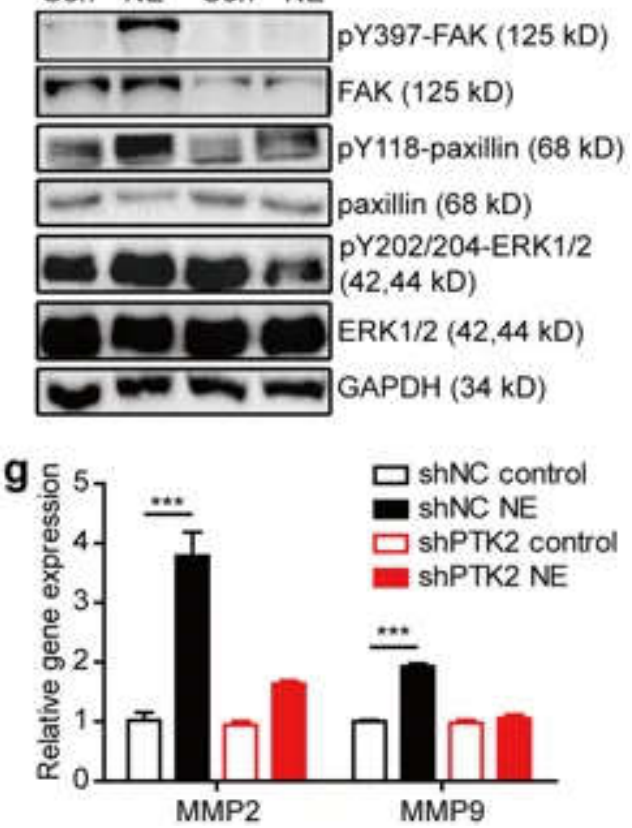

Norepinephrine promotes prostate cancer cell migration and invasion mediated by FAK. a Cell migration assay of PC-3 cells in the presence of $10 \mu \mathrm{M}$ norepinephrine, $10 \mu \mathrm{M}$ PF562 271 or 10 $\mu \mathrm{M}$ PF562 271 and $10 \mu \mathrm{M}$ norepinephrine for $36 \mathrm{~h}$. Bar: $500 \mu \mathrm{m}$. b Cell invasion assay of PC-3 cells in the presence of $10 \mu \mathrm{M}$ norepinephrine or, $10 \mu \mathrm{M}$ PF562 271 or $10 \mu \mathrm{M}$ PF562 271 and $10 \mu \mathrm{M}$ norepinephrine for $24 \mathrm{~h}$. Bar: $500 \mu \mathrm{m}$. c Cell migration assay of shNC and shPTK2 expressing PC-3 cells in the presence of $10 \mu \mathrm{M}$ norepinephrine. Bar: $500 \mu \mathrm{m}$. d Cell invasion assay of shNC and shPTK2 expressing PC-3 cells in the presence of $10 \mu \mathrm{M}$ norepinephrine. Bar: $500 \mu \mathrm{m}$. e Staining of pY118 paxillin with anti-pY118 paxilin antibody and F-actin with phalloidin of shNC and ShPTK2 expressing PC-3 cells incubated with $10 \mu \mathrm{M}$ norepinephrine for $18 \mathrm{~h}$. Bar: $5 \mu \mathrm{m}$. f Western blot 
analysis of shNC and shPTK2 expressing PC-3 cells treated with $10 \mu \mathrm{M}$ norepinephrine for 10 minutes. g MMP2 and MMP9 mRNA of shNC and shPTK2 expressing PC-3 cells after incubation with $10 \mu \mathrm{M}$ norepinephrine for $6 \mathrm{~h}$ assessed by qRT-PCR. The results were representative of three independent experiments conducted in triplicate

\section{Norepinephrine activation of FAK is mediated by $\beta 2 A R-c A M P-P K A$ pathway}

We investigated the effect of norepinephrine on FAK phosphorylation, and detected strongly increased phosphorylation of FAK Tyr397 during 2-h treatments with norepinephrine reaching maximum at 10 min in PC-3 cells (Fig. 6a). However, we detected only minimal phosphorylation of FAK Tyr576/577 and no phosphorylation of FAK Tyr925 upon norepinephrine treatment (Supplementary Figure 7a). Similar effects were also observed in LNCaP cells (Supplementary Figure 7b). Thus, norepinephrine activates FAK mainly at Tyr397 in both androgen-dependent and independent prostate cancer cells. To identify the receptor subtype mediating norepinephrineinduced FAK activation, we treated PC-3 cells with nonspecific $\beta$ adrenergic receptor activator isoproterenol or nonspecific $\beta$ adrenergic receptor blocker propranolol for $2 \mathrm{~h}$ prior to norepinephrine exposure. We found that, similar to norepinephrine, isoproterenol induced FAK phosphorylation. Propranolol had no effect on FAK phosphorylation, but blocked norepinephrineinduced FAK activation (Fig. 6b). We also examined the expression of beta-adrenergic receptors in the cell lines we used. We found no significant difference of each beta-adrenergic receptor between different cell lines, however, $\beta 2$ adrenergic receptor ( $\beta 2 \mathrm{AR})$ levels were much higher than $\beta 1 \mathrm{AR}$ and $\beta 3 A R$ (Supplementary Figures 5c-h). We found that the $\beta 2 A R$-specific blocker ICI118 551 completely blocked norepinephrine-induced FAK activation, however the $\beta 1$ AR-selective blocker atenolol only partially blocked this effect (Fig. 6c, d). 
Fig. 6
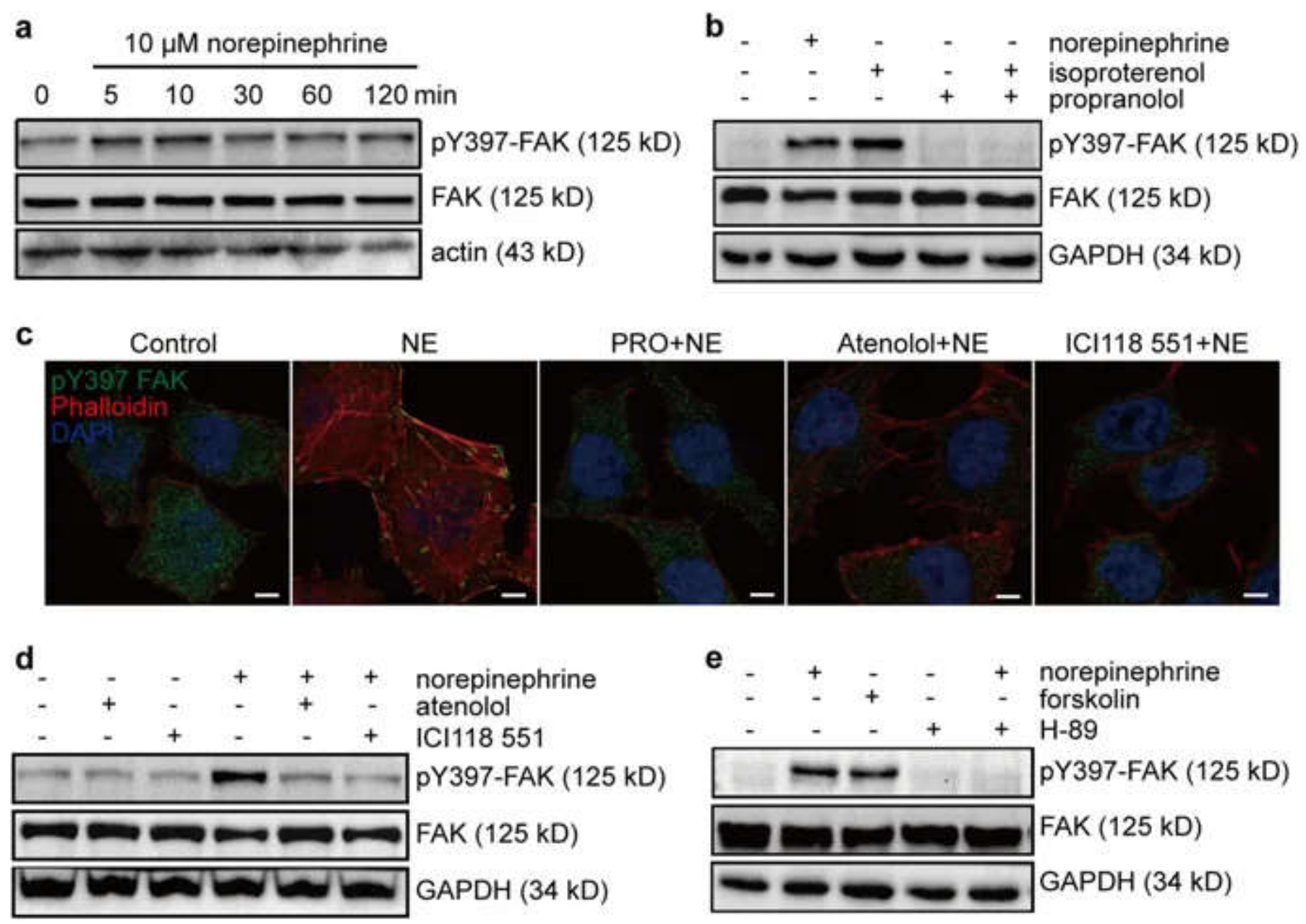

norepinephrine

atenolol

ICl118 551

pY397-FAK (125 kD)

FAK (125 kD)

GAPDH (34 kD)

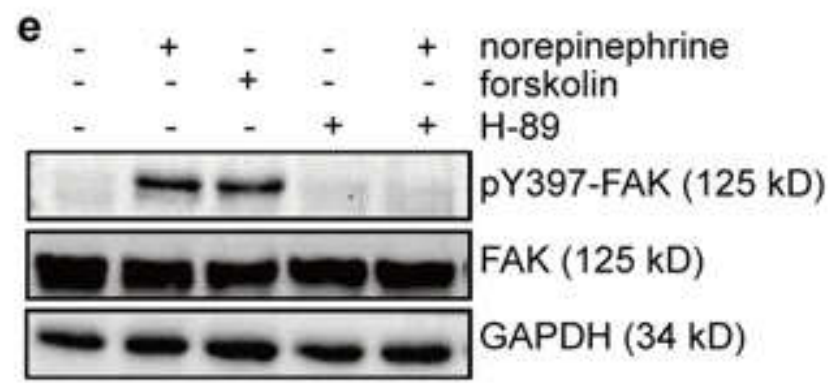

Norepinephrine-induced activation of FAK is mediated by the $32 A R-C A M P-P K A$ pathway. a Western blot analysis of FAK phosphorylation in PC-3 cells treated with norepinephrine for different times. b Western blot analysis of PC-3 cells treated with $10 \mu \mathrm{M}$ norepinephrine, $10 \mu \mathrm{M}$ isoproterenol for $10 \mathrm{~min}$ and $10 \mu \mathrm{M}$ propranolol $2 \mathrm{~h}$ before $10 \mu \mathrm{M}$ norepinephrine treatment for 10 min in the presence of propranolol. c Immunofluorescence staining of pY397 FAK and F-actin treated with $10 \mu \mathrm{M}$ norepinephrine for $18 \mathrm{~h}$ and $10 \mu \mathrm{M}$ propranolol, $10 \mu \mathrm{M}$ atenolol or $10 \mu \mathrm{M}$ ICI118 $5512 \mathrm{~h}$ before $10 \mu \mathrm{M}$ norepinephrine treatment for $18 \mathrm{~h}$ in the presence of propranolol, atenolol or ICI118 551 respectively. Bar: $5 \mu \mathrm{m}$. d Western blot analysis of PC-3 cells treated with $10 \mu \mathrm{M}$ norepinephrine for $10 \mathrm{~min}$, or $10 \mu \mathrm{M}$ atenolol or $10 \mu \mathrm{M}$ ICI118 $5512 \mathrm{~h}$ before $10 \mu \mathrm{M}$ norepinephrine treatment in the presence of atenolol or ICI118,551 respectively. e Western blot analysis of PC-3 cells treated with $10 \mu \mathrm{M}$ forskolin for $10 \mathrm{~min}$, or treated with $10 \mu \mathrm{M} \mathrm{H}-892 \mathrm{~h}$ before $10 \mu \mathrm{M}$ norepinephrine treatment in the presence of $\mathrm{H}-89$. The experiments were performed at least three times independently 
The $\beta 2 \mathrm{AR}$ activated by norepinephrine is known to activate the cAMP/PKA pathway, which can lead to phosphorylation of tyrosine kinases. To determine whether the activation of FAK by $\beta 2 A R$ was mediated by cAMP/PKA pathway, we examined the effects of forskolin, an adenylyl cyclase activator and H-89, a PKA inhibitor on FAK activation. We found that forskolin significantly increased FAK phosphorylation, whereas H-89 abolished norepinephrine-induced FAK activation (Fig. 6e). Collectively, these results show that norepinephrine activates FAK through the $32 \mathrm{AR}-$ cAMP-PKA signaling pathway. There are several pathways that lead from increased cAMP levels and PKA activation to phosphorylation of tyrosines [27], including Tyr phosphorylation by PKA itself [28]. Which of these, or others are involved in prostate cancer invasion, remains for future studies.

\section{Discussion}

Since the publication of a pioneering study showing the importance of psychological and behavioral dimensions of illness [29], many studies have focused on the effects of psychological distress on tumor progression [30,31]. To foster the clinical implications in cancer treatments, we looked for degrees of psychological depression in 98 prostate cancer patients in order to identify the precise characteristics of cancer that depression might impact. Here, we provided evidence that instead of affecting primary tumor growth, depression mainly promotes tumor invasion into the vessels and metastasis in lymph gland or lung. We found that FAK was activated in prostate cancer patients with high depression score. Recently, FAK inhibitors, such as PF562 271 and VS-4718, are in clinical development (www.clinicaltrials.gov NCT00666926, NCT01849744). FAK is a nonreceptor protein tyrosine kinase that is over-expressed and activated in several advanced-stage solid cancers [32]. The best-characterized mechanism for FAK activation involves integrin receptor clustering upon cell binding to ECM proteins [33]. However, whether FAK can be affected by depression was largely uncertain. Early studies have identified adrenergic dependent activation of FAK in ovarian cancer [34] and renal cell carcinoma [35]. Using the Hi-myc mouse model, we found depression to significantly increase sympathetic nerve infiltrations and elevated norepinephrine levels in the prostate. For the first time, we detected FAK activation in tumor 
samples of patients with high depression scores and in the prostates of stressed WT and Hi-myc mice. Furthermore, in cell line experiments, we demonstrated that norepinephrine activates FAK via the $\beta 2 A R-c A M P-P K A$ signaling pathway. Genetic or pharmaceutical inhibition of FAK blocked norepinephrine-induced cytoskeleton dynamics and ECM remolding. Taken together, our studies in multiple cell lines, animal models, and primary prostate cancer samples suggest that (i) Depressionpromoted prostate cancer invasion is mediated by FAK activation; (ii) FAK activation during depression induces an invasive phenotype of prostate cancer via regulation of the cell's cytoskeleton and remolding of the ECM by release of MMP2 and/or MMP9; (iii) FAK activation during depression is mediated by the sympathetic-cAMP signaling pathway (Fig. 7). 
Fig. 7

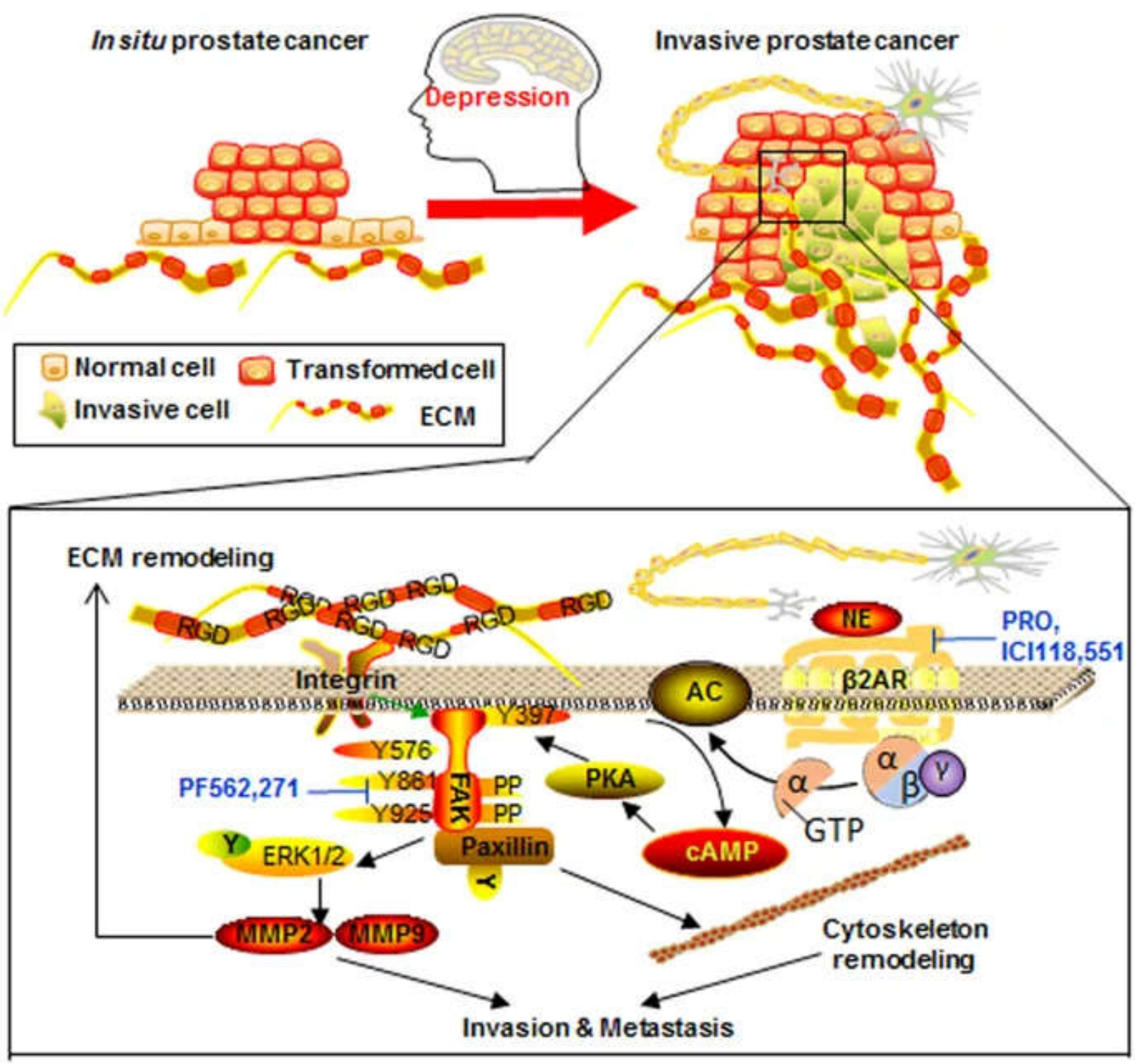

The sympathetic-cAMP-FAK signaling pathway mediates psychological depression-induced prostate cancer invasion and metastasis. Depression induces signaling from the sympathetic nerve and increases levels of norepinephrine, which facilitates prostate cancer invasion and metastasis by FAK activation. The activation of FAK further mediates paxillin-induced cytoskeleton remodeling and MMP-induced ECM remodeling, resulting in a vicious circle of FAK activation. These effects may be blocked using $\beta 2 A R$ inhibitors propranolol (PRO) and ICI118 551, and FAK inhibitor PF562 271 
Because the nervous system can process environmental stress and transmit signals to the whole body, the regulatory roles of nervous system in immunity [36, 37] and hematopoiesis [38] have long been studied. Many studies have reported that depression promotes tumor progression by altering the tumor immunity [39] or vasculature [31, 40]. Recently, another tumor microenvironment factor, desmoplasia, i.e., increased production of dense ECM around tumor cells, is reported to play a pivotal role in tumor progression [41]. However, whether depression can alter tumor fibroblasts and ECM have remained largely unknown. Our findings provide new mechanistic insight into the role of ECM remodeling in depression-induced cancer invasion. We found depression to contribute to sympathetic-FAK signaling promoting MMP2 and/or MMP9 release into the tumor environment, thus remodeling ECM. Studies have reported that norepinephrine can induce fibrosis in systemic sclerosis [42]. Similarly, we detected increased fibroblasts and collagens in the stressed Hi-myc mice compared to non-stressed Hi-myc mice. So, whether and how depression may directly affect tumor desmoplasia needs further investigation.

Our finding that depression significantly increased nerve infiltration into prostate cancer provided new evidence for the active role of nerve in tumor progression. Two landmark publications, one in prostate [6] and the other in gastric [43] cancers, have demonstrated the direct stimulating role of nerves in cancer progression by surgically cutting afferent nerves, or injection of neurotoxic drugs. Before that, nerves were considered as bystanders [44] or as a conduit for tumor perineural invasion [45]. Using NF-L to mark newly formed nerves, we found that the newly formed nerve fibers were increased more than 2-fold in stressed mice compared to non-stressed mice. Although studies have shown that tumor cells can release nerve growth factors that promote nerve-genesis in the tumor microenvironment [46], to our understanding, we found for the first time that psychological depression can stimulate nerve-genesis in the prostate. Further studies need to investigate whether depression can induce nerve-genesis in other organs. Recent studies reported that chronic stress can enlarge lymphatic vessels [31]. Besides, in tumor angiogenesis, the newly formed vessels are curved and fragile [47]. So, new techniques need to be applied in defining the characteristics of nerve fibers in the tumor environment, especially in depression situation.

Besides SNS activation, depression can also induce the activation of the hypothalamic-pituitary- 
adrenal (HPA) axis. Our findings showed that glucocorticoids were elevated $20 \%$ in the prostate of stressed mice compared with non-stressed mice (Supplementary Figure 4d). Glucocorticoids have been used in combination with chemotherapy in men with metastatic prostate cancer for decades to prevent adverse reactions. However, glucocorticoids may also activate glucocorticoid receptor (GR) or mutated androgen receptor (AR) and promote the occurrence of castration-resistant prostate cancer (CRPC) [48]. We speculated glucocorticoids may also promote depression-induced prostate cancer invasion. However, we found no changes upon dexamethasone treatment (Supplementary Figures 8a-c). In a concurrent study, a randomized phase 2 trial of dexamethasone versus prednisolone in CRPC shows that both dexamethasone and prednisolone are well tolerated in treating men with advanced prostate cancer [49]. Several points should be highlighted here. First, the concentration of glucocorticoids in depression and in clinical use in the prostate may not be enough to activate GR or mutant AR, because we found no changes of PSA levels between patients with low or high depression levels, and in non-stressed versus stressed mice. Second, patients participating in the clinical trials with combined use of glucocorticoids are already in advanced prostate cancer or CRPC, so drug resistance may still occur independently of glucocorticoid use.

It is important to notice that exercise has been shown to increase epinephrine, to mobilize NK cells and to suppress inoculated B16F10 melanoma growth [50], which contributes to the ambiguous effects of sympathetic signaling in cancer. As depression is a type of chronic stress, exercise can be regarded as an acute stress. The concentration and duration of elevated epinephrine and/or norepinephrine are relatively smaller and shorter in chronic stress than acute stress. A relatively small increase in epinephrine is sufficient to mobilize NK cells [51]. In addition, the treatment duration in their work is much shorter. Apart from that, neither subcutaneous inoculation nor tail vein injection of B16F10 melanoma cells rely on the direct regulation of nervous system when compared to spontaneous tumor or orthotopic transplanted tumor models. Other studies show that activation of PGC-1 $\alpha 1$-PPAR $\alpha / \delta$ pathway during exercise prevents depression by reducing kynurenine to cross the blood-brain barrier [52]. Taken together, the sympathetic signaling plays different roles in exercise and depression. It is plausible that both exercise and reduction of depression can inhibit tumor growth. 
In conclusion, we found FAK activated by sympathetic-cAMP-FAK signaling is pivotal for depression-promoted prostate cancer invasion. Although systemic regulation of the nervous system may bring complicated effects to the whole body, local regulation of its downstream signaling molecule FAK may provide a better opportunity to prevent and treat the invasive prostate cancer.

\section{Materials and methods}

\section{Clinical investigation}

A total of 98 prostate cancer patients were studied at Jiangsu Province Hospital of Traditional Chinese Medicine. For evaluation of patient's depression, a rating scale was established by combining the Hamilton Depression Scale (his-rating) and the PHQ-9 Depression Scale (selfrating). The median score among all patients was considered as the boundary for dividing high depression and low depression patients. The studies were conducted in accordance with International Ethical Guidelines for Biomedical Research Involving Human Subjects (CIOMS), with the approval of Jiangsu Province Hospital of Traditional Chinese Medicine (Nanjing, China). All patients provided written informed consent.

\section{Reagents and antibodies}

Norepinephrine (A7257), propranolol (P0884), and atenolol (A7655) were purchased from SigmaAldrich (St. Louis, MO, USA). Isoproterenol (1129810) from Aladdin (Shanghai, China), ICI118 551 (S8114), forskolin (S2449), PF-562 271 (S2890, formula: C21H20F3N7O3S) from Selleckchem (Houston, TX, USA). H-89 (S1644) from Beyotime (Nantong, Jiangsu, China).

Anti-neurofilament light (NF-L) (AB9568) was purchased from Millipore (Germany); antipY397FAK (ab81298), anti- $\alpha$-SMA (ab5694), anti-pY118Paxillin (ab75740) from Abcam (Cambridge, MA, USA); FAK antibody sampler kit (\#9330), anti-pY118Paxillin (\#2541), antiPaxillin (\#2542), anti-ERK (\#4695), anti-p42/44ERK (\#4370), anti-MMP2 (\#40994), anti-MMP9 (\#13667) from Cell Signaling Technology (Beverly, MA, USA); Alexa Fluor 633 conjugated 
phalloidin (A22284), goat anti-rabbit IgG $(\mathrm{H}+\mathrm{L})$ secondary antibody (Alexa Fluor 488 conjugate; R37116) from Thermo Fisher (USA).

\section{Cell culture and shRNA knockdown}

PC-3 cells (Cobioer, Nanjing, China) were cultured in F12 Medium, 22Rv1 cells (Cobioer, Nanjing, China) and LNCaP cells (Cell bank of Chinese Academy of Sciences, Shanghai, China) were cultured in RPMI-1640 Medium. All cell lines were cultured in medium supplemented with $10 \%$ fetal bovine serum (Sigma) and $1 \%$ Penicillin-Streptomycin (Gibico) at $37^{\circ} \mathrm{C}$ in a humidified $5 \% \mathrm{CO}_{2}$ incubator. All cell lines were authenticated by the STR (Short Tandem Repeat) profiling and tested for and confirmed a lack of mycoplasma using GMyc-PCR Mycoplasma Test Kit (YeSen, Shanghai, China; 40601ES10). sh-hPTK2-Luc-Puro and sh-negative control (NC)-LucPuro lentiviruses were purchased from HanBio Technology Co. Ltd. (HanBio, Shanghai, China). Lentivirus infections were performed according to the manufactures' instructions.

\section{Animals and stress procedures}

For the orthotopic xenograft model, BALB/c nude mice (6 weeks old) purchased form Charles River (Beijing, China) were allocated into groups of equal average baseline body weight and baseline sucrose intake in sucrose preference test (SPT) prior to chronic mild unpredictable stress (CUMS) treatments. Chronic stress procedure was performed as described [30, 31, 40]. Briefly, stressed mice were restrained for $2 \mathrm{~h}$ every day for 2 weeks before tumor transplantation, and continued to be restrained daily for 5 weeks. Xenograft tumors were monitored by bioluminescence images acquired using an IVIS Spectrum imaging system at the Nanjing Medical University Animal Center. For transgenic animal experiments, the Hi-myc mice (FVB-Tg (ARR2/Pbsn- MYC) 7Key, strain number: 01XK8) were obtained from the Mouse Repository of the National Cancer Institute, USA [53]. Mice were genotyped by PCR for Myc presence using mouse genomic DNA from tail biopsies. Stressed mice were housed singly and subjected to CUMS paradigm 
(Supplementary Table 2). All animal experiments were performed in accordance with the National Institutes of Health Guide for the Care and Use of Laboratory Animals, with the approval of Center for New Drug Evaluation and Research, China Pharmaceutical University (Nanjing, China). Sample sizes for animal in each group were determined based on previous studies in nu/nu mice and Hi-myc mice $[6,31,40]$ for obtaining enough samples for protein extraction, RNA extraction, and pathological examination.

\section{Behavior tests}

Investigators were blinded to group allocation when assessing animal behaviors. During the SPT, mice were given access to water and $1 \%$ sucrose solution at the same time. The preference for sucrose was measured as a percentage of the consumed sucrose solution relative to the total amount of liquid intake. During tail suspension test (TST), mice were individually suspended by the distal portion of their tails with adhesive tape for a period of $6 \mathrm{~min}$. The duration of immobility was scored for the last $4 \mathrm{~min}$. During forced swim test (FST), each mouse was placed in a cylinder container containing $20 \mathrm{~cm}$ of water. The duration of immobility was scored for the last $4 \mathrm{~min}$ in a 6-min period.

\section{Histologic staining}

Investigators were blinded to group allocation in animal tissue pathological examination. For histologic examination, the prostate tissue from each animal were fixed in neutral $10 \%$ formalin for $24 \mathrm{~h}$, embedded in paraffin and cut into 4- $\mu \mathrm{m}$-thick sections, then stained with H\&E. Masson's Trichrome staining kits (Catalog number: D026) were purchased from Nanjing Jiancheng Bioengineering Institute and performed according to manufacturer's instructions. Immunohistochemistry and immunoflurocence were performed as previously described [54]. 


\section{Western blot}

Western blot was performed as previously described [54].

\section{Quantitative PCR}

Quantitative PCR was performed as previously described [55]. Primer sequences were listed in Supplementary Table 3.

\section{Wound-healing and cell invasion assays}

For the wound-healing assay, confluent prostate cell monolayers were wounded by scratching a pipette tip from the top to the bottom of the well. Then images were taken of the whole length of the wound at the indicated time points using a Leica DMI 3000 B microscope. For cell invasion assay, cells in media containing 2\% FBS were seeded into the top chamber of an $8 \mu \mathrm{m}$ pore transwell insert (Corning, NY) coated with Matrigel (BD Biosciences) $\left(1 \times 10^{4}\right.$ cells/well/100 $\mu$ l) in a 24-well plate. Medium containing 20\% FBS was added to the bottom chamber and cells were allowed to migrate for indicated time. Cells that had not migrated were wiped from the top of the chamber. The chambers were fixed with 10\% TCA, stained with $0.4 \%$ SRB (Sulforhodamine B) (Sigma, USA) for 30 min and washed five times with $1 \%$ acetic acid. Images were taken using a Leica DMI 3000 B microscope.

\section{Statistical analysis}

All data analysis in this study was carried out using GraphPad Prism 5 or SPSS Statistics 19. All data represent multiple independent experiments conducted in triplicate and were represented as mean \pm SEM unless otherwise indicated. Statistical analyses were indicated in the figure legends. 
The association between depression levels and clinical and pathologic outcomes was calculated using chi-square correlations and multivariable Cox regression analysis. $P \leq 0.05$ was considered statistically significant. The data met the assumptions of the tests. No samples or animals were excluded from the analysis.

\section{References}

1. Mehta RD, Roth AJ. Psychiatric considerations in the oncology setting. CA Cancer J Clin. 2015;65:300-14.

2. Lopez-Calderero I, Lopez-Fando L, Rios-Gonzalez E, Maisonobe P, Hernandez-Yuste E, Sarmiento-Jordan M. Impact of locally advanced or metastatic prostate cancer on the quality of life. Actas Urol Esp. 2017;41:368-75.

3. Prasad SM, Eggener SE, Lipsitz SR, Irwin MR, Ganz PA, Hu JC. Effect of depression on diagnosis, treatment, and mortality of men with clinically localized prostate cancer. J Clin Oncol. 2014;32:2471-8.

4. Batty GD, Russ TC, Stamatakis E, Kivimaki M. Psychological distress in relation to site specific cancer mortality: pooling of unpublished data from 16 prospective cohort studies. BMJ 2017;356:j108.

5. Sullivan DR, Forsberg CW, Ganzini L, Au DH, Gould MK, Provenzale D, et al. Longitudinal changes in depression symptoms and survival among patients with lung cancer: a national cohort assessment. J Clin Oncol. 2016;34:3984-91. 
6. Magnon C, Hall SJ, Lin J, Xue X, Gerber L, Freedland SJ, et al. Autonomic nerve development contributes to prostate cancer progression. Science. 2013;341:1236361.

7. Ayala GE, Dai H, Powell M, Li R, Ding Y, Wheeler TM, et al. Cancer-related axonogenesis and neurogenesis in prostate cancer. Clin Cancer Res. 2008;14:7593-603.

8. Lu D, Sinnott JA, Valdimarsdottir U, Fang F, Gerke T, Tyekucheva S, et al. Stress-related signaling pathways in lethal and nonlethal prostate cancer. Clin Cancer Res. 2016;22:765-72.

9. Kupfer DJ, Frank E, Phillips ML. Major depressive disorder: new clinical, neurobiological, and treatment perspectives. Lancet. 2012;379:1045-55.

10. Gundem G, Van Loo P, Kremeyer B, Alexandrov LB, Tubio JM, Papaemmanuil E, et al. The evolutionary history of lethal metastatic prostate cancer. Nature. 2015;520:353-7.

11. Harryman WL, Hinton JP, Rubenstein CP, Singh P, Nagle RB, Parker SJ, et al. The cohesive metastasis phenotype in human prostate cancer. Biochim Biophys Acta. 2016;1866:221-31.

12. Gaston SM, Soares MA, Siddiqui MM, Vu D, Lee JM, Goldner DL, et al. Tissue-print and print-phoresis as platform technologies for the molecular analysis of human surgical specimens: mapping tumor invasion of the prostate capsule. Nat Med. 2005;11:95-101.

13. de la Taille A, Rubin MA, Buttyan R, Olsson CA, Bagiella E, Burchardt M, et al. Is microvascular invasion on radical prostatectomy specimens a useful predictor of PSA recurrence for prostate cancer patients? Eur Urol. 2000;38:79-84. 
14. Malik R, Lelkes PI, Cukierman E. Biomechanical and biochemical remodeling of stromal extracellular matrix in cancer. Trends Biotechnol. 2015;33:230-6.

15. Ko CJ, Huang CC, Lin HY, Juan CP, Lan SW, Shyu HY, et al. Androgen-induced TMPRSS2 activates matriptase and promotes extracellular matrix degradation, prostate cancer cell invasion, tumor growth, and metastasis. Cancer Res. 2015;75:2949-60.

16. Mo F, Lin D, Takhar M, Ramnarine VR, Dong $X$, Bell RH, et al. Stromal gene expression is predictive for metastatic primary prostate cancer. Eur Urol. 2017:e-pub ahead of print 2017; doi:2010.1016/j.eururo.2017.2002.2038.

17. Nader GP, Ezratty EJ, Gundersen GG. FAK, talin and PIPKIgamma regulate endocytosed integrin activation to polarize focal adhesion assembly. Nat Cell Biol. 2016;18:491-503.

18. Slack-Davis JK, Hershey ED, Theodorescu D, Frierson HF, Parsons JT. Differential requirement for focal adhesion kinase signaling in cancer progression in the transgenic adenocarcinoma of mouse prostate model. Mol Cancer Ther. 2009;8:2470-7.

19. Feng $Y$, Huang $W$, Tian TF, Wang G, Hu C, Chiu HF, et al. The psychometric properties of the Quick Inventory of Depressive Symptomatology-Self-Report (QIDS-SR) and the Patient Health Questionnaire-9 (PHQ-9) in depressed inpatients in China. Psychiatry Res. 2016;243:92-6.

20. Furukawa TA. Assessment of mood: guides for clinicians. J Psychosom Res. 2010;68:581-9.

21. Katz RJ. Animal model of depression: pharmacological sensitivity of a hedonic deficit. Pharmacol, Biochem, Behav. 1982;16:965-8. 
22. Willner P, Towell A, Sampson D, Sophokleous S, Muscat R. Reduction of sucrose preference by chronic unpredictable mild stress, and its restoration by a tricyclic antidepressant. Psychopharmacology. 1987;93:358-64.

23. Hill MN, Hellemans KG, Verma P, Gorzalka BB, Weinberg J. Neurobiology of chronic mild stress: parallels to major depression. Neurosci Biobehav Rev. 2012;36:2085-117.

24. Cole SW, Sood AK. Molecular pathways: beta-adrenergic signaling in cancer. Clin Cancer Res. 2012;18:1201-6.

25. Barbieri A, Bimonte S, Palma G, Luciano A, Rea D, Giudice A, et al. The stress hormone norepinephrine increases migration of prostate cancer cells in vitro and in vivo. Int J Oncol. $2015 ; 47: 527-34$.

26. Lopez-Colome AM, Lee-Rivera I, Benavides-Hidalgo R, Lopez E. Paxillin: a crossroad in pathological cell migration. J Hematol Oncol. 2017;10:50.

27. Law NC, Donaubauer EM, Zeleznik AJ, Hunzicker-Dunn M. How protein kinase A activates canonical tyrosine kinase signaling pathways to promote granulosa cell differentiation. Endocrinology. 2017;158:2043-51.

28. Piiper A, Lutz MP, Cramer H, Elez R, Kronenberger B, Dikic I, et al. Protein kinase A mediates cAMP-induced tyrosine phosphorylation of the epidermal growth factor receptor. Biochem Biophys Res Commun. 2003;301:848-54.

29. Engel GL. The need for a new medical model: a challenge for biomedicine. Science. 1977;196:129-36. 
30. Hassan S, Karpova Y, Baiz D, Yancey D, Pullikuth A, Flores A, et al. Behavioral stress accelerates prostate cancer development in mice. J Clin Invest. 2013;123:874-86.

31. Le CP, Nowell CJ, Kim-Fuchs C, Botteri E, Hiller JG, Ismail H, et al. Chronic stress in mice remodels lymph vasculature to promote tumour cell dissemination. Nat Commun. $2016 ; 7: 10634$.

32. Sulzmaier FJ, Jean C, Schlaepfer DD. FAK in cancer: mechanistic findings and clinical applications. Nat Rev Cancer. 2014;14:598-610.

33. Jin JK, Tien PC, Cheng CJ, Song JH, Huang C, Lin SH, et al. Talin 1 phosphorylation activates beta1 integrins: a novel mechanism to promote prostate cancer bone metastasis. Oncogene. 2015;34:1811-21.

34. Sood AK, Armaiz-Pena GN, Halder J, Nick AM, Stone RL, Hu W, et al. Adrenergic modulation of focal adhesion kinase protects human ovarian cancer cells from anoikis. J Clin Invest. 2010;120:1515-23.

35. Ma X, Zhao Y, Daaka Y, Nie Z. Acute activation of beta2-adrenergic receptor regulates focal adhesions through betaArrestin2- and p115RhoGEF protein-mediated activation of RhoA. J Biol Chem. 2012;287:18925-36.

36. Pavlov VA, Tracey KJ. Neural regulation of immunity: molecular mechanisms and clinical translation. Nat Neurosci. 2017;20:156-66.

37. Glaser R, Kiecolt-Glaser JK. Stress-induced immune dysfunction: implications for health. Nat Rev Immunol. 2005;5:243-51. 
38. Madden KS. Sympathetic neural-immune interactions regulate hematopoiesis, thermoregulation and inflammation in mammals. Dev Comp Immunol. 2017;66:92-7.

39. Qin J-f, Jin F-j, Li N, Guan H-t, Lan L, Ni H. et al. Adrenergic receptor $\beta 2$ activation by stress promotes breast cancer progression through macrophages M2 polarization in tumor microenvironment. BMB Rep. 2015;48:295-300.

40. Thaker PH, Han LY, Kamat AA, Arevalo JM, Takahashi R, Lu C, et al. Chronic stress promotes tumor growth and angiogenesis in a mouse model of ovarian carcinoma. Nat Med. 2006;12:939-44.

41. Incio J, Liu H, Suboj P, Chin SM, Chen IX, Pinter M, et al. Obesity-induced inflammation and desmoplasia promote pancreatic cancer progression and resistance to chemotherapy. Cancer Discov. 2016;6:852-69.

42. Uehara A, Motegi S, Yamada K, Uchiyama A, Perera B, Toki S, et al. Mechanistic insight into the norepinephrine-induced fibrosis in systemic sclerosis. Sci Rep. 2016;6:34012

43. Zhao CM, Hayakawa Y, Kodama Y, Muthupalani S, Westphalen CB, Andersen GT, et al. Denervation suppresses gastric tumorigenesis. Sci Transl Med. 2014;6:250ra115.

44. Batkin S, Piette LH, Wildman E. Effect of muscle denervation on growth of transplanted tumor in mice. Proc Natl Acad Sci USA. 1970;67:1521-7.

45. Marchesi F, Piemonti L, Mantovani A, Allavena P. Molecular mechanisms of perineural invasion, a forgotten pathway of dissemination and metastasis. Cytokine Growth Factor Rev. 2010;21:77-82. 
46. Venkatesh H, Monje M. Neuronal activity in ontogeny and oncology. Trends Cancer. 2017;3:89-112.

47. Jayson GC, Kerbel R, Ellis LM, Harris AL. Antiangiogenic therapy in oncology: current status and future directions. Lancet. 2016;388:518-29.

48. Arora VK, Schenkein E, Murali R, Subudhi SK, Wongvipat J, Balbas MD, et al. Glucocorticoid receptor confers resistance to antiandrogens by bypassing androgen receptor blockade. Cell. 2013;155:1309-22.

49. Venkitaraman R, Lorente D, Murthy V, Thomas K, Parker L, Ahiabor R, et al. A randomised phase 2 trial of dexamethasone versus prednisolone in castration-resistant prostate cancer. Eur Urol. 2015;67:673-9.

50. Pedersen L, Idorn M, Olofsson GH, Lauenborg B, Nookaew I, Hansen RH, et al. Voluntary running suppresses tumor growth through epinephrine- and IL-6-dependent NK cell mobilization and redistribution. Cell Metab. 2016;23:554-62.

51. Dimitrov S, Lange T, Born J. Selective mobilization of cytotoxic leukocytes by epinephrine. J Immunol. 2010;184:503-11.

52. Agudelo LZ, Femenia T, Orhan F, Porsmyr-Palmertz M, Goiny M, Martinez-Redondo V, et al. Skeletal muscle PGC-1alpha1 modulates kynurenine metabolism and mediates resilience to stress-induced depression. Cell. 2014;159:33-45. 
53. Ellwood-Yen K, Graeber TG, Wongvipat J, Iruela-Arispe ML, Zhang J, Matusik R, et al. Myc-driven murine prostate cancer shares molecular features with human prostate tumors. Cancer Cell. 2003;4:223-38.

54. Mao J, Wang D, Wang Z, Tian W, Li X, Duan J, et al. Combretastatin A-1 phosphate, a microtubule inhibitor, acts on both hepatocellular carcinoma cells and tumor-associated macrophages by inhibiting the Wnt/beta-catenin pathway. Cancer Lett. 2016;380:134-43.

55. Li X, Wang D, Chen Z, Lu E, Wang Z, Duan J, et al. Galphai1 and Galphai3 regulate macrophage polarization by forming a complex containing CD14 and Gab1. Proc Natl Acad Sci USA. 2015;112:4731-6.

\section{Acknowledgements}

We thank Mrs. Ning Su for IHC studies, Mrs. Jian-hua Ding for HPLC studies, and Dr. Wen-tao Liu for gelatin zymography experiments and helpful suggestions. This study was supported by grants from Intramural Research Program of the NIH (Z01-ES101684 to LB), The 111 Project (No.111-2-07), National Science Foundation of China (91529304, 81673468 to YY, 81603132 to XG and 81403020 to LY), Natural Science Foundation of Jiangsu Province (No. BK20160753 to XG), and China Postdoctoral Science Foundation (No. 2016M591965 to XG).

\section{Author information}

These authors contributed equally: Yan Cheng, Xing-Hua Gao, Xian-Jing Li.

These authors jointly supervised this work: Lutz Birnbaumer, Yong Yang.

\section{Affiliations}


State Key Laboratory of Natural Medicines, Jiangsu Key Laboratory of Drug Discovery for

Metabolic Disease, Center for New Drug Safety Evaluation and Research, China Pharmaceutical

University, Nanjing, 211198, China

Yan Cheng, Xing-Hua Gao, Xian-Jing Li, Qiu-Hua Cao, Dan-Dan Zhao, Hong-Xi Wu, Yun Wang, LinJun You, Hong-Bao Yang, Yun-Long He, Yong-Ren Li \& Yong Yang

Nutrition/Metabolism Laboratory, Department of Surgery, Beth Israel Deaconess Medical Center, Harvard Medical School, Boston, MA, 02138, USA

Jin-Rong Zhou

Department of Pharmacology, Yong Loo Lin School of Medicine, National University of Singapore, Singapore, 117597, Singapore

Jin-Song Bian

Department of Urology, Jiangsu Province Hospital of Traditional Chinese Medicine, Nanjing, 210029, China

Qing-Yi Zhu

Neurobiology Laboratory, National Institute of Environmental Health Sciences, Durham, NC, 27709, USA

Lutz Birnbaumer

Biomedical Research Institute,(BOMED), Catholic University of Argentina, Buenos Aires, C1107AAZ, Argentina

Lutz Birnbaumer

\section{Corresponding authors}

Correspondence to Lutz Birnbaumer or Yong Yang.

\section{Ethics declarations}

\section{Conflict of interest}


The authors declare that they have no conflict of interest.

\section{Electronic supplementary material}

Supplementary Figure 1 - https://static-content-springer-com.ezproxy.nihlibrary.nih.gov /esm/art\%3A10.1038\%2Fs41388-018-0177-4/MediaObjects 141388_2018_177_MOESM1_ESM.tif

Supplementary Figure 2 - https://static-content-springer-com.ezproxy.nihlibrary.nih.gov lesm/art\%3A10.1038\%2Fs41388-018-0177-4/MediaObjects 141388_2018_177_MOESM2_ESM.tif

Supplementary Figure 3 - https://static-content-springer-com.ezproxy.nihlibrary.nih.gov lesm/art\%3A10.1038\%2Fs41388-018-0177-4/MediaObjects 141388_2018_177_MOESM3_ESM.tif

Supplementary Figure 4 - https://static-content-springer-com.ezproxy.nihlibrary.nih.gov /esm/art\%3A10.1038\%2Fs41388-018-0177-4/MediaObjects 141388_2018_177_MOESM4_ESM.tif

Supplementary Figure 5 - https://static-content-springer-com.ezproxy.nihlibrary.nih.gov lesm/art\%3A10.1038\%2Fs41388-018-0177-4/MediaObjects 141388_2018_177_MOESM5_ESM.tif

Supplementary Figure 6 - https://static-content-springer-com.ezproxy.nihlibrary.nih.gov lesm/art\%3A10.1038\%2Fs41388-018-0177-4/MediaObjects 141388_2018_177_MOESM6_ESM.tif

Supplementary Figure 7 - https://static-content-springer-com.ezproxy.nihlibrary.nih.gov lesm/art\%3A10.1038\%2Fs41388-018-0177-4/MediaObjects 141388_2018_177_MOESM7_ESM.tif

Supplementary Figure 8 - https://static-content-springer-com.ezproxy.nihlibrary.nih.gov /esm/art\%3A10.1038\%2Fs41388-018-0177-4/MediaObjects 141388_2018_177_MOESM8_ESM.tif 
Supplementary Figure Legends - https://static-content-springer-com.ezproxy.nihlibrary.nih.gov lesm/art\%3A10.1038\%2Fs41388-018-0177-4/MediaObjects 141388_2018_177_MOESM9_ESM.docx

Supplementary Table 1 - https://static-content-springer-com.ezproxy.nihlibrary.nih.gov lesm/art\%3A10.1038\%2Fs41388-018-0177-4/MediaObjects 141388_2018_177_MOESM10_ESM.doc

Supplementary Table 2 - https://static-content-springer-com.ezproxy.nihlibrary.nih.gov /esm/art\%3A10.1038\%2Fs41388-018-0177-4/MediaObjects 141388_2018_177_MOESM11_ESM.docx

Supplementary Table 3 - https://static-content-springer-com.ezproxy.nihlibrary.nih.gov lesm/art\%3A10.1038\%2Fs41388-018-0177-4/MediaObjects 141388_2018_177_MOESM12_ESM.docx

\section{Rights and permissions}

Reprints and Permissions

\section{About this article}




\section{Cite this article}

Cheng, Y., Gao, X., Li, X. et al. Depression promotes prostate cancer invasion and metastasis via a sympathetic-CAMP-FAK signaling pathway. Oncogene 37, 2953-2966 (2018) doi:10.1038/s41388-018-0177-4

Received

Revised

Accepted

25 September 2017

21 December 2017

29 January 2018

Published

Issue Date

08 March 2018

31 May 2018

DOI

https://doi-org.ezproxy.nihlibrary.nih.gov/10.1038/s41388-018-0177-4

\section{Share this article}

Anyone you share the following link with will be able to read this content:

Get shareable link

\section{Subjects Prostate cancer - Targeted therapies}

\section{Further reading}

$\beta 3$-Adrenoceptor as a potential immuno-suppressor agent in melanoma - https://doiorg.ezproxy.nihlibrary.nih.gov/10.1111/bph.14660

Maura Calvani, Gennaro Bruno[...] Luca Filippi

British Journal of Pharmacology (2019) 


\section{ature a

SPRINGER NATURE
(C) 2019 Springer Nature Limited

SPRINGER NATURE
(C) 2019 Springer Nature Limited .

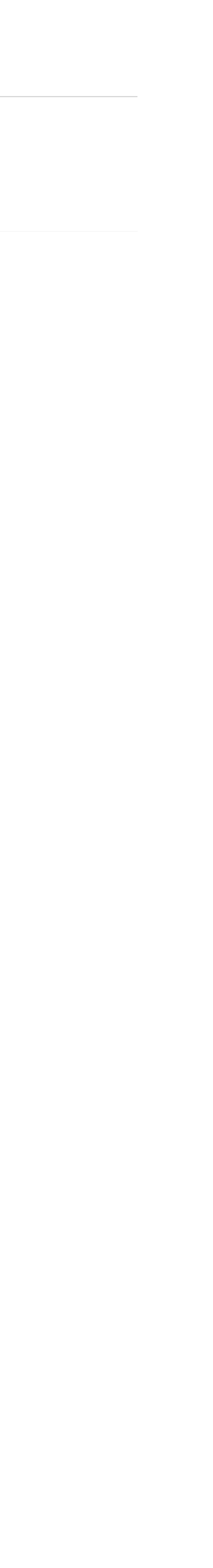

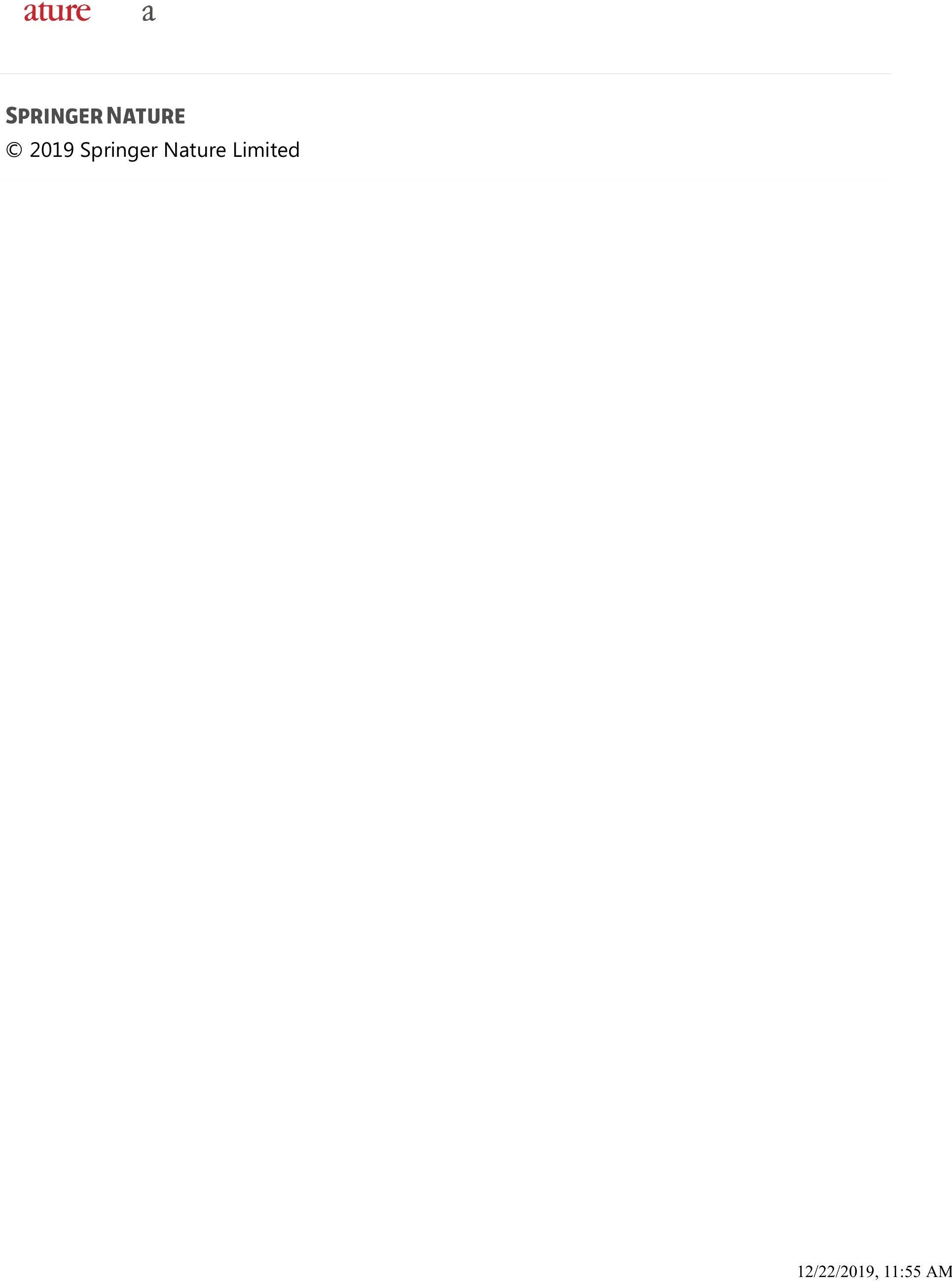

